\title{
Three-body force for baryons from the D0-D4/D8 matrix model
}

\author{
Si-wen L: 1 and Tuo Jid 2 \\ Department of Modern Physics, \\ University of Science and Technology of China, \\ Hefei 230026, Anhui, China
}

\begin{abstract}
This is an extensive work to our previous paper [8] studied on the D0-D4/D8 holographic system. We compute the three-body force for baryons with the D0-D4/D8 matrix model derived in [8] with considering the non-zero QCD vacuum. We obtain the three-body force at short distances but modified by the appearance of the smeared D0-branes i.e. considering the effects from the non-trivial QCD vacuum. We firstly test our matrix model in the case of 't Hooft instanton and then in two more realistic case: (1) three-neutrons with averaged spins and (2) proton-proton-neutron (or proton-neutron-proton). The three-body potential vanishes in the former case while in two latter cases it is positive i.e. repulsive and makes sense only if the constraint for stable baryonic state is satisfied. We require all the baryons in our computation aligned on a line. These may indicate that the cases in dense states of neutrons such as in neutron stars, Helium-3 or Tritium nucleus all with the non-trivial QCD vacuum.
\end{abstract}

\footnotetext{
${ }^{1}$ Email: cloudk@mail.ustc.edu.cn

${ }^{2}$ Email: jt2011@mail.ustc.edu.cn
} 


\section{Introduction}

In nuclear physics, there is one of the fundamental ingredients which is how to describe the interaction among nucleons. QCD as an underlying theory of strong interactions with asymptotic freedom, makes it possible to form the confinement such as bound states of nucleons. People have to study on the behavior of nucleons to describe the nuclear force. As it is known, the nuclear force can not only be explained by two-body force, but also three-body force which plays the important role as well. For example, the three-body nuclear force is vital in studying on the excitation spectra of light nuclei or high-density baryon matters such as supernovae or neutron stars. However the properties of three-body nuclear force are still to be revealed although it has been developed so many years. Since QCD at nonzero baryon density is strongly coupled which thus is notoriously difficult to solve, consequently it becomes the main obstacle for revealing the aspects of nuclear force.

On the other hand, some classical or semi-classical gauge field configurations may also be important in QCD or nuclear physics, for example, some topologically nontrivial solutions such as instantons, monopoles and so on. In [1, 2, 3, 4, the self-dual field strength has been studied and proposed to be a mechanism for the confinement [5]. Therefore, the states with non-zero QCD vacuum $\theta$ angle (or equivalently non-zero $\theta \operatorname{Tr}\left(F_{\mu \nu} \tilde{F}^{\mu \nu}\right)$, here $\tilde{F}^{\mu \nu}$ is the dual field of the gauge field strength $F_{\mu \nu}$.) could exist and affect the the mass of meson with the interaction among baryons, and this $\theta$-dependent term may also lead to some other observable effects such as in the glueball condensation or the chiral magnetic effect (CME) 6, 7]. Thus in this paper, we would like to study on the three-body nuclear force with non-zero QCD vacuum $\theta$ angle by using the D0-D4/D8 holographic matrix model proposed in [8] for baryons. We compute the three-body force at short distances for baryons in the large $N_{c}$ holographic QCD explicitly while the two-body case has already been studied in [8].

By holography, in order to describe the states with non-zero $\operatorname{Tr}\left(F_{\mu \nu} \tilde{F}^{\mu \nu}\right)$ in the dual field theory, it corresponds to adding smeared D0-branes to the compacted D4-brane background [9, 10]. And with introducing the flavor $\mathrm{D} 8 / \overline{\mathrm{D} 8}$-branes, the meson spectrum has been studied in [9] while the baryon spectrum was studied in [11] with the approach of Yang-Mills instanton. As a comparison with [11] and study from [12], we use the gauge/string duality (or AdS/CFT correspondence, see [13, 14, 15] for a review.) to derive our matrix model from the Sakai-Sugimoto model [16, 17] in the D0-D4 background [9] (i.e. D0-D4/D8 system), in the large- $N_{c}$ limit at a large 't Hooft coupling $\lambda$. That is to say, our matrix model is also a low-energy effective theory on the baryon vertex, which is the D4'-brane 3 wrapped on $S^{4}$ in D0-D4/D8 system [9, 18, in the gravity side of gauge/string duality.

The matrix model could describe $k$-body baryons with arbitrary $k$, since the rank of the matrix is determined by the value of $k$, where $k$ is the baryon number. In our matrix model, the positions of $k$ baryons are represented by the diagonal elements of the matrices after integrating out the off-diagonal elements. Furthermore, the classical values of a pair of the complex $k \times N_{f}$ rectangular matrices are related to the sizes of baryons and they describe the dynamics of the strings connecting the flavor D8/D8-branes and the baryon vertices. With all of these, it comes to the well-known Atiyah-Drinfeld-Hitchin-Manin (ADHM) matrix model of instantons.

In our previous work [8], we also studied on the baryon spectrum $(k=1)$ and two-body force at short distances for baryons $(k=2)$, except the derivation of our matrix model. For the case of $k=1$, we find a constraint for stable baryonic state in D0-D4/D8 system which is exactly the same as the conclusion in [11] with the approach of Yang-Mills instanton, but quite different from the original Sakai-Sugimoto model.

\footnotetext{
${ }^{3}$ In order to distinguish the D4-branes which are responsible for the background geometry, we use D4'-brane to denote the baryon vertex since in D0-D4/D8 system the baryon vertex is also a D4-brane.
} 
And our baryon spectrum could fit the experimental data well just by adjusting the number density of the D0-branes. For the case of $k=2$, we have studied on the two-body force at short distances and also found a universal repulsive core for any baryonic state but modified by the appearance of the smeared D0-branes. It turns out that the two-body force at short distances could become attractive which describes an unstable two-body system if the constraint for stable baryonic state is overcome.

Instead of phenomenological models, our matrix model is for multi-bayon systems with non-zero QCD vacuum $\theta$ angle and based on the gauge/string duality with the underlying string theory, so it is natural and interesting to extend the analysis to derive the three-body force in D0-D4/D8 system with our matrix model. So in this paper, we extend our previous work in [8] and continue the analysis to the case of $k=3$, as a parallel computation to [19, to study the three-body force at short distances with non-trivial QCD vacuum by using our matrix model. We will focus on the two particular examples which are three neutrons with averaged spins and proton-proton-neutron (or proton-neutron-neutron), and require all the baryons or nucleons aligned on a line with equal spacings for each case. The system with averaged spins is typical for dense states of multi-baryons in QCD, such as cores of neutron stars, while the latter one is related to tritium nuclei or Helium-3. From our results, we find the three-body potential is suppressed if compared to the two-body force in [8]. And both in the case of averaged spins and proton-proton-neutron, the three-body potential would be totally complex if $\zeta=U_{Q_{0}}^{3} / U_{K K}^{3}>2$ where $U_{Q_{0}}^{3}$ is related to the number density of the smeared D0-brane. This result is exactly the same as the constraint for the stable baryonic state claimed in [8] and [1] with the approach of Yang-Mills instanton.

In this paper, the organization is as follows. In section 2, we briefly review the D0-D4/D8 matrix model and the calculations for two-body force as shown in [8]. In section 3, we calculate the three-body force with the "classical treatment" i.e. the case with spin or isospin aligned classically. In this case we find a vanished three-body force which is independent on the non-zero QCD vacuum $\theta$ anglet, however this result is similar as in [19] and consistent with the soliton approach in [20. Then we employ the set-up for generic three-body forces with quantum spin/isospin, the resultant three-body force is modified by the appearance of the smeared D0-branes (or equivalently by considering the non-trivial QCD vacuum) and also consistent with the constraint for stable baryonic states claimed in [8, 11. The summary and discussion are in the final section.

\section{A brief review of D0-D4/D8 matrix model}

To calculate the three-body force for baryons by using D0-D4/D8 matrix model is quite analogous to the computation of the two-body force which has been performed in [8]. In this section, we will give a brief summary of the D0-D4/D8 matrix model and the calculations of the two-body force for baryons with this model.

\subsection{Action of D0-D4/D8 matrix model}

We proposed a D0-D4/D8 matrix model in [8] by using the standard technique in string theory from the Sakai-Sugimoto model in the D0-D4 background (i.e. D0-D4/D8 system). It is a quantum mechanical system with $U(k)$ symmetry which takes the following action

\footnotetext{
${ }^{4}$ In fact, our result depends on the parameter $\zeta$ in this holographic model, however it has been turned out $\zeta$ is related to the parameter $\theta$ in the topological term in QCD 9 .
} 


$$
\begin{aligned}
S= & \frac{\lambda N_{c} M_{K K}}{54 \pi}(1+\zeta)^{3 / 2} \operatorname{Tr} \int d t\left[\left(D_{0} X^{M}\right)^{2}-\frac{2}{3}\left(1-\frac{1}{2} \zeta\right) M_{K K}^{2}\left(X^{4}\right)^{2}\right. \\
& +D_{0} \bar{\omega}_{i}^{\dot{\alpha}} D_{0} \omega_{i \dot{\alpha}}-\frac{1}{6}\left(1-\frac{1}{2} \zeta\right) M_{K K}^{2} \bar{\omega}_{i}^{\dot{\alpha}} \omega_{i \dot{\alpha}} \\
& \left.+\frac{3^{6} \pi^{2}}{4 \lambda^{2} M_{K K}^{4}} \frac{1}{(1+\zeta)^{4}}(\vec{D})^{2}+\vec{D} \cdot \vec{\tau}_{\dot{\beta}}^{\dot{\alpha}} \bar{X}^{\dot{\beta} \alpha} X_{\dot{\alpha} \alpha}+\vec{D} \cdot \vec{\tau}_{\dot{\beta}}^{\dot{\alpha}} \bar{\omega}^{\dot{\beta} \alpha} \omega_{\dot{\alpha} \alpha}\right] \\
& +N_{c} \operatorname{Tr} \int d t A_{0} .
\end{aligned}
$$

It is allowed to change the baryon number $k$ by choosing the gauge group $U(k)$ for the matrix model (2.1) to describe $k$-body interaction in the D0-D4/D8 system. Note that the rank of the gauge group $U(k)$ is the number of baryons. The parameter $\lambda=g_{Y M}^{2} N_{c}$ is the 't Hooft coupling constant and $M_{K K}$ is a unique scale while the parameter $\zeta$ is $\zeta=U_{Q_{0}}^{3} / U_{K K}^{3}$, where $U_{Q_{0}}^{3}$ is related to the number density of smeared D0-branes. $N_{c}$ and $N_{f}$ represent the number of colors and flavors respectively. We have obtained the baryon spectrum for the case of $k=1$ and fitted the experimental data by adjusting the parameter $\zeta$, computed the two-body force (i.e. $k=2$ ) for baryons at short distances with the matrix model (2.1) in [8]. To clarify the symmetry in the matrix model (2.1), we summarize the field content in the following table.

\begin{tabular}{|c|c|c|c|c|}
\hline Fields & index & $U(k)$ & $S U\left(N_{f}\right)$ & $S U(2) \times S U(2)$ \\
\hline \hline$X^{M}$ & $M=1,2,3,4$ & adj & 1 & $(2,2)$ \\
\hline$\omega_{i \dot{\alpha}}$ & $\dot{\alpha}=1,2 ; i=1,2 \ldots N_{f}$ & adj & fund & $(1,2)$ \\
\hline$A_{0}$ & & adj & 1 & $(1,1)$ \\
\hline$D_{s}$ & $s=1,2,3$ & 1 & 1 & $(1,3)$ \\
\hline
\end{tabular}

Table 1: Fields in the matrix model

$A_{0}$ and $\vec{D}$ are auxiliary fields while $X$ and $\omega$ are dynamical fields. For a more realistic case and simplicity, only the two-flavor case is considered throughout this paper, i.e. $N_{f}=2$. In the action (2.1), the trace is taken over the indices of $U(k)$ group. The total symmetry of the matrix model (2.1) is $U(k) \times S U\left(N_{f}\right) \times S O(3)$, where the first $U(k)$ group is a local symmetry group while the last $S O(3)$ represents the spatial rotation group which forms a broken $S O(4) \simeq S U(2) \times S U(2)$ in the holographic dimension as shown in the table. The broken symmetry yields the mass terms of $X^{4}$ and $\omega$. The covariant derivatives in action (2.1) are defined as $D_{0} X^{M}=\partial_{0} X^{M}-i\left[A_{0}, X^{M}\right], D_{0} \omega=\partial_{0} \omega-i A_{0} \omega$ and $D_{0} \bar{\omega}=\partial_{0} \bar{\omega}+i A_{0} \bar{\omega}$. The indices of spinor for $X$ are defined as $X_{\alpha \dot{\alpha}}=\left(X^{M} \sigma_{M}\right)_{\alpha \dot{\alpha}}$ and $\sigma_{M}=(i \vec{\tau}, 1), \bar{\sigma}_{M}=(-i \vec{\tau}, 1)$ where $\vec{\tau}$ 's are Pauli matrices since only two-flavor case $\left(N_{f}=2\right)$ is the concern. Other details about this matrix model from the Sakai-Sugimoto model in the D0-D4 background are in [8].

\subsection{Two-body effective force for baryons from D0-D4 matrix model}

Let us explain briefly how to calculate the two-body effective force for baryons at short distances from the matrix model (2.1) (See [8] for the complete review), and it is also a parallel computation to [12]. We first obtain the two-body Hamiltonian by integrating out the auxiliary field $A_{0}$ and describe a single baryon by its wave function. However, the key here is to solve the "ADHM constraint" 21] to minimize the potential 
introduced after integrating out the other auxiliary field $\vec{D}$. Since only two-flavor case $\left(N_{f}=2\right)$ is the concern, the ADHM constraint could be written exactly as

$$
\vec{\tau}_{\dot{\beta}}^{\dot{\alpha}}\left(\bar{X}^{\dot{\beta} \alpha} X_{\dot{\alpha} \alpha}+\bar{\omega}^{\dot{\beta} \alpha} \omega_{\dot{\alpha} \alpha}\right)_{B A}=0
$$

with the indices $A, B=1,2 \ldots k$.

The equation (2.2) could be solved by chosen $\omega_{\dot{\alpha} i}=U_{\dot{\alpha} i} \rho$ for the case of a single baryon, where $U$ is a $S U(2)$ matrix. For the two-body case (i.e. $k=2$ ), the generic solution could be chosen as the ADHM data of $S U$ (2) Yang-Mills instantons, which are

$$
X^{M}=\tau^{3} \frac{r_{M}}{2}+\tau^{1} Y_{M}, \omega_{\dot{\alpha} i}^{A=1}=U_{\dot{\alpha} i}^{A=1} \rho_{1}, \omega_{\dot{\alpha} i}^{A=2}=U_{\dot{\alpha} i}^{A=2} \rho_{2},
$$

where $Y^{M}$ is the off-diagonal part of $X^{M}$ which is defined as

$$
Y_{M}=-\frac{\rho_{1} \rho_{2}}{4\left(r_{L}\right)^{2}} \operatorname{Tr}\left[\bar{\sigma}_{M} r_{N} \sigma_{N}\left(\left(U^{1}\right)^{\dagger} U^{2}-\left(U^{2}\right)^{\dagger} U^{1}\right)\right] .
$$

We define $|r|^{2}=\left(r^{M}\right)^{2}$ and the vector $r^{M}$ is interpreted as the distance between the two baryons. $U^{(1)}$ and $U^{(2)}$ are all $S U(2)$ matrices as the moduli parameters for each instanton while $\rho_{1}$ and $\rho_{2}$ are associated with the size of each instantons. The ADHM constraint is satisfied with this choice and the potential associated with $\vec{D}$ in the action (2.1) vanishes.

With the decomposition of $U(2) \simeq U(1) \times S U(2)$, i.e. $A_{0}=A_{0}^{0} \mathbf{1}_{2 \times 2}+A_{0}^{1} \tau^{1}+A_{0}^{2} \tau^{2}+A_{0}^{3} \tau^{3}$, it is straightforward to obtain the two-body Hamiltonian after integrating out the auxiliary field $A_{0}$ to evaluate the terms with $A_{0}$ in the action (2.1),

$$
\begin{aligned}
S_{\text {kinetic }+C S}^{\text {on-shell }}= & \frac{\lambda N_{c} M_{K K}}{54 \pi}(1+\zeta)^{3 / 2} \operatorname{Tr} \int d t\left[\left(D_{0} X^{M}\right)^{2}+D_{0} \bar{\omega}_{i}^{\dot{\alpha}} D_{0} \omega_{i \dot{\alpha}}\right]+N_{c} \operatorname{Tr} \int d t A_{0} \\
= & \frac{\lambda N_{c} M_{K K}}{54 \pi}(1+\zeta)^{3 / 2} \int d t\left\{2\left(A_{0}^{1}\right)^{2} r_{M}^{2}+8\left(A_{0}^{3}\right)^{2} Y_{M}^{2}\right. \\
& +2\left(\rho_{1}^{2}+\rho_{2}^{2}\right)\left[\left(A_{0}^{0}\right)^{2}+\left(A_{0}^{1}\right)^{2}+\left(A_{0}^{3}\right)^{2}\right] \\
& \left.+4 \rho_{1} \rho_{2} A_{0}^{0} A_{0}^{1} \operatorname{Tr}\left[\left(U^{1}\right)^{\dagger} U^{2}\right]+4\left(\rho_{1}^{2}-\rho_{2}^{2}\right) A_{0}^{0} A_{0}^{3}+\frac{108 \pi}{\lambda M_{K K}}(1+\zeta)^{-3 / 2} A_{0}\right\} .
\end{aligned}
$$

We need to substitute the solutions for all the components of $A_{0}$ back into (2.5) once we solve the equations of motion for $A_{0}$. The potential could be evaluated by using $\int d t V=-S_{\text {on-shell }}$ as

$$
\begin{aligned}
V & =2 V_{1-\text { body }}+V_{2-\text { body }}, \quad V_{1-\text { body }}=\frac{27 \pi N_{c}}{4 \lambda M_{K K}} \frac{1}{(1+\zeta)^{3 / 2}}, \\
V_{2-\text { body }} & =\frac{27 \pi N_{c}}{\lambda M_{K K}} \frac{1}{(1+\zeta)^{3 / 2}} \frac{u_{0}^{2}}{|r|^{2}+2 \rho^{2}-2 u_{0}^{2} \rho^{2}} .
\end{aligned}
$$

Here we have used the same notation as [19] by defining $u_{0}=\frac{1}{2}\left(\operatorname{Tr}\left[\left(U^{1}\right)^{\dagger} U^{2}\right]\right)$ with the choice of $\rho_{1}=\rho_{2}=\rho$ and kept the leading term in the large $N_{c}$ expansion only.

There is also an additional term to (2.5) which is the mass term for $X^{4}$ in the action (2.1), 


$$
\begin{aligned}
& \frac{\lambda N_{c} M_{K K}}{54 \pi}(1+\zeta)^{3 / 2} \frac{2}{3}\left(1-\frac{1}{2} \zeta\right) M_{K K}^{2} \operatorname{Tr}\left(X^{4}\right)^{2} \\
= & \frac{\lambda N_{c} M_{K K}}{81 \pi}(1+\zeta)^{3 / 2}\left(1-\frac{1}{2} \zeta\right) M_{K K}^{2}\left(\frac{r_{4}^{2}}{2}+Y_{4}^{2}\right) .
\end{aligned}
$$

Thus there is an additional two-body potential from the off-diagonal components of $Y$ which is

$$
V_{2-\text { body }}^{\text {mass }}=\frac{\lambda N_{c} M_{K K}}{162 \pi}(1+\zeta)^{3 / 2}\left(1-\frac{1}{2} \zeta\right) M_{K K}^{2}\left[\frac{\rho_{1}^{2} \rho_{2}^{2}}{\left(r_{M}^{2}\right)^{2}}\left(r_{i} \operatorname{Tr}\left[i \tau^{i} U^{(1) \dagger} U^{(2)}\right]\right)^{2}\right]
$$

with $i=1,2,3$. So we have the total two-body potential which is the sum of (2.6) and (2.8). Note that the four-dimensional inter-baryon distance $|r|^{2}$ is equal to the distance between baryons in three dimensions, since for the leading order in the large $N_{c}$ expansion, the classical value of the $X^{4}$ vanishes for the single instantons.

Finally, in order to evaluate the vacuum expectation of the potential (2.6) and (2.8), we need to use the nucleon wave function as in [8, 12, 19, 22], which is

$$
\frac{1}{\pi}\left(\tau^{2} U\right)_{I J}=\left(\begin{array}{ll}
\mid p \uparrow> & \mid p \downarrow> \\
\mid n \uparrow> & \mid n \downarrow>
\end{array}\right)_{I J}=\frac{1}{\pi}\left(\begin{array}{cc}
a_{1}+i a_{2} & -a_{3}-i a_{4} \\
-a_{3}+i a_{4} & -a_{1}+i a_{2}
\end{array}\right)_{I J} .
$$

The $S U(2)$ matrix $U$ represents a unit 4-vector as $U=i a_{i} \tau^{i}+a_{4} \mathbf{1}_{2 \times 2}$ with the normalization $\left(a_{1}\right)^{2}+\left(a_{2}\right)^{2}+$ $\left(a_{3}\right)^{2}+\left(a_{4}\right)^{2}=1$. Using the standard definition $S_{12}=12 J_{1}^{i} \hat{r}^{i} J_{2}^{j} \hat{r}^{j}-4 J_{1}^{i} J_{2}^{i}$ with $\hat{r}^{i}=r^{i} /|r|$ and $i=1,2,3$, it yields the form $\langle V\rangle_{I_{1}, I_{2}, J_{1}, J_{2}}=V_{C}(\vec{r})+S_{12} V_{T}(\vec{r})$ as the potential of two -body nucleons. Then we obtain a central and a tensor part of the two-body force at short distances which are

$$
\begin{aligned}
V_{C}^{(0)}(\vec{r}) & =\pi\left[\frac{3^{3}}{2}+8\left(\vec{I}_{1} \cdot \vec{I}_{2}\right)\left(\vec{J}_{1} \cdot \vec{J}_{2}\right)\right] \frac{N_{c}}{\lambda M_{K K}} \frac{1}{(1+\zeta)^{3 / 2}} \frac{1}{r^{2}} \\
V_{T}^{(0)}(\vec{r}) & =2 \pi\left(\vec{I}_{1} \cdot \vec{I}_{2}\right) \frac{N_{c}}{\lambda M_{K K}} \frac{1}{(1+\zeta)^{3 / 2}} \frac{1}{r^{2}} .
\end{aligned}
$$

(2.10) is the leading order term from (2.5) in the expansion by assuming $r_{M} \gg \rho$. And we also have the next to the leading order terms in [8] which are

$$
\begin{aligned}
V_{C}^{(1)}(\vec{r}) & =\left[\frac{1}{81}-\frac{16}{2187}\left(\vec{I}_{1} \cdot \vec{I}_{2}\right)\left(\vec{J}_{1} \cdot \vec{J}_{2}\right)\right](1+\zeta)^{3 / 2}\left(1-\frac{1}{2} \zeta\right) \frac{\lambda N_{c} M_{K K}^{3}}{\pi} \frac{\rho^{4}}{r^{2}}, \\
V_{T}^{(1)}(\vec{r}) & =\frac{8}{2187}\left(\vec{I}_{1} \cdot \vec{I}_{2}\right)(1+\zeta)^{3 / 2}\left(1-\frac{1}{2} \zeta\right) \frac{\lambda N_{c} M_{K K}^{3}}{\pi} \frac{\rho^{4}}{r^{2}} .
\end{aligned}
$$

As we can see, the two-body potential is modified by the appearance of smeared D0-branes, i.e. considering the non-trivial QCD vacuum. And there would be an attractive force in (2.11) if $\zeta>2$. Thus it is just the constraint for stable baryonic states in the D0-D4/D8 system in two-body case, which is the same as the constraint for the state of the single baryonic state and is quite different from the original Sakai-Sugimoto model obviously. 


\section{Three-body force for baryons at short distances}

In this section we will test our matrix model (2.1) by computing the three-body effective potential (i.e. $k=3$ ) at short distances. It is a parallel procedure to the case of two-body potential for baryons as in the previous section, and we follow the same procedures as mentioned in [19] which are

A. Choose the value of $k$ (the number of baryons), and solve the ADHM constraint (i.e. integrate out the auxiliary field $\vec{D}$ and minimize the ADHM potential).

B. Substitute the solution back into the action (2.1) of the matrix model.

C. Integrate out the auxiliary field $A_{0}$.

D. Evaluate the Hamiltonian with the desired baryonic state.

As a first test, we will consider a case that all three baryons take the same classical spin or isospin. And secondly, we will demonstrate the computations for baryons which are aligned on a straight line with equal spacings after taking the explicit set-up for generic quantum spin or isospin to our system. In fact we are also less clear about how to get a physical interpretation from the calculations for the baryons with generic positions, thus we also choose this linear position to simplify and illuminate our calculations. Finally, we finish our tests by evaluating the three-body Hamiltonian with two specific three-body quantum states. They are the states of three neutrons with averaged spins and proton-proton-neutron (or proton-neutron-proton).

\subsection{Three-body force for baryons with classical spin/isospin}

Our goal is to evaluate the three-body Hamiltonian, so let us start with the four steps mentioned above.

\section{A. Solve the ADHM constraint}

First, we need to consider minimizing the ADHM potential in the matrix model. This is equivalent to solve the ADHM constraint for any $A, B=1,2,3$. A simple solution to this constraint is

$$
\begin{aligned}
\omega_{\dot{\alpha} i}^{A} & =U_{\dot{\alpha} i} \rho^{A}, \quad(A=1,2,3) \\
X^{M} & =\sum_{a=3,8} \frac{\lambda^{a}}{2} r_{a}^{M} .
\end{aligned}
$$

(3.1) is nothing but the ADHM data for the 't Hooft instantons which has been used in [8, 12, 19]. Noting that the $2 \times 2$ unitary matrix $U$ does not depend on the index $A$ while the degrees of freedom $\omega$ correspond to the spin and isospin. The matrices $X$ are diagonal and their diagonal elements represent the location of the baryons with $M=1,2,3$. The $X$ 's and special $\omega$ 's of the ADHM data for the 't Hooft instantons (3.1) are sharing the same orientation. "Classical spin/isospin" here means that in fact we can not fix the orientation $U$ and consider the wave functions with finite width at same time. All the terms with the commutators $[X, X]$ vanish since the matrices $X$ are diagonal, which obviously satisfies the ADHM constraint. 


\section{B. Substitute the ADHM data to the matrix action}

The inter-baryon potential comes from the terms after integrating out the auxiliary field $A_{0}$ and the mass term of $X^{4}$. However the mass term of $X^{4}$ vanishes for the 't Hooft instantons because of no off-diagonal components in (3.1). And on the other hand, we are going to choose the gauge $\partial_{0} \omega_{\dot{\alpha} i}^{A}=0$, thus there is no time dependence in $\omega$ or $X$. So in this section, we need to consider the terms related to $A_{0}$ only.

With the gauge group $U(k)$ and $k=3$ for three-body case, the auxiliary field $A_{0}$ could be written exactly by the Gell-Mann matrices $\lambda^{a}$ which is

$$
A_{0}=A_{0}^{0} \mathbf{1}_{3 \times 3}+\sum_{a=1}^{8} A_{0}^{a} \frac{\lambda^{a}}{2} .
$$

As in the two-body case, only the kinetic terms of $\omega$ 's and $X$ 's contain $A_{0}$ while only $U(1)$ component $A_{0}^{0}$ appears in the CS term in action (2.1). So for the kinetic term of $X$, it takes the following exact forms by substituting the ADHM data (3.1),

$$
\begin{aligned}
\operatorname{Tr}\left(D_{0} X^{M}\right)^{2}= & \frac{1}{2}\left[\left(A_{0}^{1} r_{3}^{M}\right)^{2}+\left(A_{0}^{2} r_{3}^{M}\right)^{2}\right]+\frac{1}{8}\left[\left(A_{0}^{4}\right)^{2}+\left(A_{0}^{5}\right)^{2}\right]\left(r_{3}^{M}+\sqrt{3} r_{8}^{M}\right)^{2} \\
& +\frac{1}{8}\left[\left(A_{0}^{6}\right)^{2}+\left(A_{0}^{7}\right)^{2}\right]\left(r_{3}^{M}-\sqrt{3} r_{8}^{M}\right)^{2}
\end{aligned}
$$

And then we need to consider the kinetic term for $\omega$. It is a lengthy but straightforward calculation just by using the ADHM data (3.1). As a result, we obtain the following expression,

$$
\begin{aligned}
\operatorname{Tr}\left(D_{0} \bar{\omega}_{i}^{\dot{\alpha}} D_{0} \omega_{i \dot{\alpha}}\right)= & 2\left[\left(\rho^{1}\right)^{2}+\left(\rho^{2}\right)^{2}+\left(\rho^{3}\right)^{2}\right]\left[\left(A_{0}^{0}\right)^{2}+\frac{1}{6} \sum_{a=1}^{8}\left(A_{0}^{a}\right)^{2}\right]+4 \rho^{1} \rho^{2} A_{0}^{1} A_{0}^{0}+4 \rho^{1} \rho^{3} A_{0}^{4} A_{0}^{0} \\
& +4 \rho^{2} \rho^{3} A_{0}^{6} A_{0}^{0}+2 A_{0}^{3} A_{0}^{0}\left[\left(\rho^{1}\right)^{2}-\left(\rho^{2}\right)^{2}\right]+\frac{2}{\sqrt{3}} A_{0}^{8} A_{0}^{0}\left[\left(\rho^{1}\right)^{2}+\left(\rho^{2}\right)^{2}-2\left(\rho^{3}\right)^{2}\right] \\
& +\frac{2 \rho^{1} \rho^{2}}{\sqrt{3}} A_{0}^{1} A_{0}^{8}+\rho^{1} \rho^{2} A_{0}^{4} A_{0}^{6}+\rho^{1} \rho^{2} A_{0}^{5} A_{0}^{7}-\frac{\rho^{1} \rho^{3}}{\sqrt{3}} A_{0}^{4} A_{0}^{8}+\rho^{1} \rho^{3} A_{0}^{1} A_{0}^{6}-\rho^{1} \rho^{3} A_{0}^{2} A_{0}^{7} \\
& +\rho^{1} \rho^{3} A_{0}^{3} A_{0}^{4}-\frac{\rho^{2} \rho^{3}}{\sqrt{3}} A_{0}^{6} A_{0}^{8}+\rho^{2} \rho^{3} A_{0}^{1} A_{0}^{4}+\rho^{2} \rho^{3} A_{0}^{2} A_{0}^{5}-\rho^{2} \rho^{3} A_{0}^{3} A_{0}^{6} \\
& +\left[\frac{1}{\sqrt{3}} A_{0}^{3} A_{0}^{8}+\frac{1}{4}\left(A_{0}^{4}\right)^{2}+\frac{1}{4}\left(A_{0}^{5}\right)^{2}-\frac{1}{4}\left(A_{0}^{6}\right)^{2}-\frac{1}{4}\left(A_{0}^{7}\right)^{2}\right]\left[\left(\rho^{1}\right)^{2}-\left(\rho^{2}\right)^{2}\right] \\
& +\frac{1}{12}\left[2\left(A_{0}^{1}\right)^{2}+2\left(A_{0}^{2}\right)^{2}+2\left(A_{0}^{3}\right)^{2}-2\left(A_{0}^{8}\right)^{2}-\left(A_{0}^{4}\right)^{2}-\left(A_{0}^{5}\right)^{2}-\left(A_{0}^{6}\right)^{2}-\left(A_{0}^{7}\right)^{2}\right] \\
& \times\left[\left(\rho^{1}\right)^{2}+\left(\rho^{2}\right)^{2}-2\left(\rho^{3}\right)^{2}\right] .
\end{aligned}
$$

In fact (3.3) and (3.4) are as same as the "data" used in [19] since we start with the same ADHM data (3.1) for the 't Hooft instantons.

And finally, we also need to consider the Lagrangian for CS term which is

$$
L_{C S}=\frac{162 \pi}{\lambda M_{K K}(1+\zeta)^{3 / 2}} A_{0}^{0} .
$$


So the total Lagrangian involving the field $A_{0}$ consists of (3.3) (3.4) and (3.5), which is denoted by $L_{A_{0}}$,

$$
L_{A_{0}}=\operatorname{Tr}\left(D_{0} X^{M}\right)^{2}+\operatorname{Tr}\left(D_{0} \bar{\omega}_{i}^{\dot{\alpha}} D_{0} \omega_{i \dot{\alpha}}\right)+L_{C S} .
$$

\section{Integrate out the auxiliary field $A_{0}$}

We need to solve all the equations of motion for all the components of $A_{0}$. All the equations of motion can be obtained by Euler-Lagrange equation with (3.6), i.e.

$$
\frac{\partial L_{A_{0}}}{\partial A_{0}^{0}}=0, \text { or } \frac{\partial L_{A_{0}}}{\partial A_{0}^{a}}=0 \quad(\text { for } \quad a=1,2 \ldots 8) .
$$

We obtain 9 equations while all the components of $A_{0}$ are mixed to each other. By solving these equations we find a unique solution a:5

\footnotetext{
${ }^{5}$ In order to simplified the formula, we have used $r_{a}^{2}$ to represent $r_{a}^{M} r_{a}^{M}$ and $r_{a} r_{b}$ to represent $r_{a}^{M} r_{b}^{M}$.
} 


$$
\begin{aligned}
& A_{0}^{0}=\frac{9 \pi}{2(1+\zeta)^{3 / 2} \lambda\left(\rho^{1}\right)^{2}\left(\rho^{2}\right)^{2}\left(\rho^{3}\right)^{2} M_{K K} r_{3}^{2}\left(r_{3}^{2}-3 r_{8}^{2}\right)^{2}} \times \\
& \left\{-r_{3}^{2}\left(\rho^{1}\right)^{2}\left(\rho^{2}\right)^{2}\left[9\left(r_{8}^{2}\right)^{2}+8 \sqrt{3} r_{3} r_{8}\left(\left(\rho^{2}\right)^{2}-\left(\rho^{1}\right)^{2}\right)\right.\right. \\
& \left.-6 r_{8}^{2}\left(r_{3}^{2}-2\left(\left(\rho^{2}\right)^{2}+\left(\rho^{1}\right)^{2}\right)\right)+r_{3}^{2}\left(r_{3}^{2}+4\left(\left(\rho^{2}\right)^{2}+\left(\rho^{1}\right)^{2}\right)\right)\right] \\
& -\left[\left(r_{3}^{2}-3 r_{8}^{2}\right)^{2}\left(\rho^{1}\right)^{2}\left(r_{3}^{2}+\left(\rho^{1}\right)^{2}\right)+\left(r_{3}^{2}-3 r_{8}^{2}\right)^{2}\left(\rho^{2}\right)^{4}\right. \\
& \left.+\left(\left(r_{3}^{2}-3 r_{3} r_{8}\right)^{2} r_{3}^{2}+18\left(r_{3}^{2}+r_{8}^{2}\right)\left(\rho^{1}\right)^{2}\right)\left(\rho^{2}\right)^{2}\right]\left(\rho^{3}\right)^{2} \\
& +4\left[2 \sqrt{3} r_{3} r_{8}\left(\left(\rho^{2}\right)^{2}-\left(\rho^{1}\right)^{2}\right)-r_{3}^{2}\left(\left(\rho^{2}\right)^{2}+\left(\rho^{1}\right)^{2}\right)-3 r_{8}^{2}\left(\left(\rho^{2}\right)^{2}+\left(\rho^{1}\right)^{2}\right)\right] \\
& \left.\times r_{3}^{2}\left(\rho^{3}\right)^{4}\right\} \text {, } \\
& A_{0}^{3}=\frac{27 \pi}{2(1+\zeta)^{3 / 2} \lambda\left(\rho^{1}\right)^{2}\left(\rho^{2}\right)^{2} M_{K K} r_{3}^{2}\left(r_{3}^{2}-3 r_{8}^{2}\right)^{2}} \times \\
& \left\{\left(r_{3}^{2}\right)^{3}\left[\left(\rho^{1}\right)^{2}-\left(\rho^{2}\right)^{2}\right]+9\left(r_{8}^{2}\right)^{2}\left[\left(\rho^{1}\right)^{4}-\left(\rho^{2}\right)^{4}\right]+\left(r_{3}^{2}\right)^{2}\left[\left(\rho^{1}\right)^{2}-\left(\rho^{2}\right)^{2}\right]\right. \\
& \times\left(\left(\rho^{1}\right)^{2}+\left(\rho^{2}\right)^{2}+4\left(\rho^{3}\right)^{2}-6 r_{8}^{2}\right)+\left[2\left(\rho^{1}\right)^{2}\left(\rho^{2}\right)^{2}+\left(\left(\rho^{1}\right)^{2}+\left(\rho^{2}\right)^{2}\right)\left(\rho^{3}\right)^{2}\right] \\
& \times 8 \sqrt{3} r_{3}^{2}\left(r_{3} r_{8}\right)+3 r_{3}^{2} r_{8}^{2}\left[\left(\rho^{1}\right)^{2}-\left(\rho^{2}\right)^{2}\right] \times \\
& \left.\left[3 r_{8}^{2}-2\left(\left(\rho^{1}\right)^{2}+\left(\rho^{2}\right)^{2}-2\left(\rho^{3}\right)^{2}\right)\right]\right\}, \\
& A_{0}^{8}=\frac{9 \pi}{2(1+\zeta)^{3 / 2} \lambda\left(\rho^{1}\right)^{2}\left(\rho^{2}\right)^{2}\left(\rho^{3}\right)^{2} M_{K K} r_{3}^{2}\left(r_{3}^{2}-3 r_{8}^{2}\right)^{2}} \times \\
& \left\{-9 \sqrt{3}\left(r_{8}^{2}\right)^{2}\left[\left(\rho^{1}\right)^{2}+\left(\rho^{2}\right)^{2}\right]^{2}\left(\rho^{3}\right)^{2}+\left[2\left(\rho^{1}\right)^{2}\left(\rho^{2}\right)^{2}-\left(\left(\rho^{1}\right)^{2}+\left(\rho^{2}\right)^{2}\right)\left(\rho^{3}\right)^{2}\right]\right. \\
& \times \sqrt{3}\left(r_{3}^{2}\right)^{3}-24 r_{3}^{2}\left(r_{3} r_{8}\right)\left[\left(\rho^{1}\right)^{2}-\left(\rho^{2}\right)^{2}\right]\left[2\left(\rho^{1}\right)^{2}\left(\rho^{2}\right)^{2}+\left(\rho^{3}\right)^{4}\right] \\
& +3 \sqrt{3} r_{3}^{2} r_{8}^{2}\left[2\left(\rho^{1}\right)^{2}\left(\rho^{2}\right)^{2}\left(3 r_{8}^{2}+4\left(\rho^{1}\right)^{2}+4\left(\rho^{2}\right)^{2}\right)-4\left(\left(\rho^{1}\right)^{2}+\left(\rho^{2}\right)^{2}\right)\left(\rho^{3}\right)^{4}\right. \\
& \left.-3 r_{8}^{2}\left(\rho^{3}\right)^{2}\left(\left(\rho^{1}\right)^{2}+\left(\rho^{2}\right)^{2}\right)+2\left(\left(\rho^{1}\right)^{4}+\left(\rho^{2}\right)^{4}+6\left(\rho^{1}\right)^{2}\left(\rho^{2}\right)^{2}\right)\left(\rho^{3}\right)^{2}\right] \\
& -\sqrt{3}\left(r_{3}^{2}\right)^{2}\left[-4\left(\rho^{1}\right)^{2}\left(\rho^{2}\right)^{2}\left(-3 r_{8}^{2}+2\left(\rho^{1}\right)^{2}+2\left(\rho^{2}\right)^{2}\right)+\left(\rho^{3}\right)^{2} \times\right. \\
& \left.\left.\left(\left(\rho^{1}\right)^{4}-6\left(\rho^{1}\right)^{2}\left(\rho^{2}\right)^{2}-6 r_{8}^{2}\left(\rho^{1}\right)^{2}-6 r_{8}^{2}\left(\rho^{2}\right)^{2}\right)+4\left(\rho^{3}\right)^{2}\left(\left(\rho^{1}\right)^{2}+\left(\rho^{2}\right)^{2}\right)\right]\right\},
\end{aligned}
$$

and 


$$
\begin{aligned}
& A_{0}^{1}=\frac{27 \pi\left[\left(\rho^{2}\right)^{2}+\left(\rho^{1}\right)^{2}\right]}{(1+\zeta)^{3 / 2} \lambda M_{K K} \rho^{1} \rho^{2} r_{3}^{2}}, \\
& A_{0}^{4}=\frac{108 \sqrt{3} \pi\left[\left(\rho^{1}\right)^{2}+\left(\rho^{3}\right)^{2}\right]}{(1+\zeta)^{3 / 2} \lambda M_{K K} \rho^{1} \rho^{3}\left[\sqrt{3} r_{3}^{2}+6 r_{3} r_{8}+3 \sqrt{3} r_{8}^{2}\right]}, \\
& A_{0}^{6}=\frac{108 \pi\left[r_{3}^{2}+2 \sqrt{3} r_{3} r_{8}+3 r_{8}^{2}\right]\left[\left(\rho^{2}\right)^{2}+\left(\rho^{3}\right)^{2}\right]}{(1+\zeta)^{3 / 2} \lambda M_{K K} \rho^{2} \rho^{3}\left(r_{3}^{2}-3 r_{8}^{2}\right)^{2}},
\end{aligned}
$$

while the solutions for other components are $A_{0}^{2}=A_{0}^{5}=A_{0}^{7}=0$. We can obtain the integrated Lagrangian from $L_{A_{0}}$ with the moduli parameters $r_{3}^{M}, r_{8}^{M}$ and $\rho^{A}$ by plugging the solutions (3.8) and (3.9) back to (3.6), which is

$$
\begin{aligned}
L_{A_{0}}\left(r_{3}^{M}, r_{8}^{M}, \rho^{A}\right)= & -\left(\frac{54 \pi}{\lambda M_{K K}}\right)^{2} \frac{1}{(1+\zeta)^{3}}\left[\frac{1}{8} \sum_{A=1}^{3} \frac{1}{\left(\rho^{A}\right)}+\frac{1}{4\left(r_{3}^{M}\right)^{2}}\left(1+\frac{\left(\rho^{1}\right)^{2}}{2\left(\rho^{2}\right)^{2}}+\frac{\left(\rho^{2}\right)^{2}}{2\left(\rho^{1}\right)^{2}}\right)\right. \\
& +\frac{1}{\left(r_{3}^{M}+\sqrt{3} r_{8}^{M}\right)^{2}}\left(1+\frac{\left(\rho^{1}\right)^{2}}{2\left(\rho^{3}\right)^{2}}+\frac{\left(\rho^{3}\right)^{2}}{2\left(\rho^{1}\right)^{2}}\right) \\
& \left.+\frac{1}{\left(r_{3}^{M}-\sqrt{3} r_{8}^{M}\right)^{2}}\left(1+\frac{\left(\rho^{2}\right)^{2}}{2\left(\rho^{3}\right)^{2}}+\frac{\left(\rho^{3}\right)^{2}}{2\left(\rho^{2}\right)^{2}}\right)\right] .
\end{aligned}
$$

By employing the picture of soliton, we obtain the potential $V^{\mathrm{cl}}$ from the D0-D4/D8 matrix model, which is

$$
S=\frac{\lambda N_{c} M_{K K}}{54 \pi}(1+\zeta)^{3 / 2} \int d t L_{A_{0}}=-\int d t V^{\mathrm{cl}} .
$$

Thus we have

$$
\begin{aligned}
V^{\mathrm{cl}}= & \frac{54 \pi N_{c}}{\lambda M_{K K}} \frac{1}{(1+\zeta)^{3 / 2}}\left[\frac{1}{8} \sum_{A=1}^{3} \frac{1}{\left(\rho^{A}\right)}+\frac{1}{4\left(r_{3}^{M}\right)^{2}}\left(1+\frac{\left(\rho^{1}\right)^{2}}{2\left(\rho^{2}\right)^{2}}+\frac{\left(\rho^{2}\right)^{2}}{2\left(\rho^{1}\right)^{2}}\right)\right. \\
& +\frac{1}{\left(r_{3}^{M}+\sqrt{3} r_{8}^{M}\right)^{2}}\left(1+\frac{\left(\rho^{1}\right)^{2}}{2\left(\rho^{3}\right)^{2}}+\frac{\left(\rho^{3}\right)^{2}}{2\left(\rho^{1}\right)^{2}}\right) \\
& \left.+\frac{1}{\left(r_{3}^{M}-\sqrt{3} r_{8}^{M}\right)^{2}}\left(1+\frac{\left(\rho^{2}\right)^{2}}{2\left(\rho^{3}\right)^{2}}+\frac{\left(\rho^{3}\right)^{2}}{2\left(\rho^{2}\right)^{2}}\right)\right] .
\end{aligned}
$$

In order to obtain the potential intrinsic to the three-body case, we have to subtract the one- and two- body Hamiltonians. It can be read from the computation for two-body case in [8] with the ADHM data for the 't Hooft instantons. If we take the leading term in the large $N_{c}$ expansion, we have the following forms

$$
V_{1-\mathrm{body}}^{\mathrm{cl}}=\frac{27 \pi N_{c}}{4 \lambda M_{K K}(1+\zeta)^{3 / 2}} \frac{1}{\left(\rho^{A}\right)^{2}}, \quad V_{2-\mathrm{body}}^{\mathrm{cl}}=\frac{27 \pi N_{c}}{4 \lambda M_{K K}(1+\zeta)^{3 / 2}} \frac{1}{\left(r^{M}\right)^{2}}\left(2+\frac{\left(\rho^{B}\right)^{2}}{\left(\rho^{A}\right)^{2}}+\frac{\left(\rho^{A}\right)^{2}}{\left(\rho^{B}\right)^{2}}\right)
$$


where we have used $r^{M}$ to represent the distance between the two baryons. Then according to (3.12) and (3.13), it gives

$$
\sum_{A=1,2,3} V_{1-\text { body }}^{(A) \mathrm{cl}}+\frac{1}{2} \sum_{A \neq B} V_{2-\text { body }}^{(A, B) \mathrm{cl}}=V^{\mathrm{cl}},
$$

which means the three-body force of the baryons sharing the same classical spins or isospins vanishes exactly. The result remains as in [19] and in [20] with the soliton approach.

In [8, 11], we claim that the constraint for the stable baryonic state is $\zeta<2$ in D0-D4/D8 system and it turns out the two-body force is also affected by this constraint. However according to (3.12) and (3.14), it seems this constraint has nothing to do with our calculations for three-body case. The direct reason is, during our calculations for the "classical treatment" we have set the mass term of $X^{4}$ to zero, but the constraint for the stable baryonic state comes from this term. The physical interpretation is, in fact we do not keep the quantum spin or isospin degrees of freedom explicitly in this "classical" computation, which means some quantum effects about the QCD vacuum in this sense is missing. Therefore the computation based on the "classical treatment" is also unfortunately unrealistic for the nucleons in the D0-D4/D8 system. So in the next sections, we will focus on a more realistic case with the quantum degrees of freedom for spin or isospin.

\subsection{Generic three-body force in D0-D4/D8 system}

For a generic calculation about the three-body baryons in D0-D4/D8 system, we will follow the four steps as well as the case in the previous section.

\section{A. Solve the ADHM constraint}

As a warm-up in the previous section, we have fixed the spins or isospins for baryons and computed the three-body force with the ADHM data for the 't Hooft instanton in our D0-D4/D8 matrix model, which is easy but not realistic. In this section, we keep the quantum spin or isospin degrees of freedom, i.e. allow arbitrary $U$ for each baryon,

$$
\omega_{\dot{\alpha} i}^{A}=U_{\dot{\alpha} i}^{A} \rho^{A}, \quad(A=1,2,3) .
$$

Since our D0-D4/D8 matrix model does not change the ADHM constraint (2.2), thus we can choose the same solution for $X$ as in [19] which is

$$
X^{M}=\sum_{a=3,8} \frac{\lambda^{a}}{2} r_{a}^{M}+\sum_{a=1,4,6} \frac{\lambda^{a}}{2} r_{a}^{M} .
$$

The off-diagonal components are turned on in matrices $X^{M}$ which makes (3.16) different from (3.1). The offdiagonal $r_{1}, r_{4}$ and $r_{6}$ should be small and $r_{3}$ and $r_{8}$ specify the positions of these three baryons. The classical size of baryon is small enough for large $\lambda, \rho \sim \lambda^{-1 / 2}$, so we also need the ADHM data for well-separated instantons since the generic three-body ADHM data is not available, i.e.

$$
\left|r_{3}+\sqrt{3} r_{8}\right| / 2,\left|-r_{3}+\sqrt{3} r_{8}\right| / 2,\left|r_{8}\right| \gg \rho^{A} .
$$

The well-separated instanton is in [23] and we employ it as the ADHM data in our notation, which the relevant parts are6

\footnotetext{
${ }^{6}$ Here we follow the notation used in [19, 24]
} 


$$
\begin{aligned}
r_{1}^{M} \sigma_{M} & =\frac{d_{12}^{M} \sigma_{M}}{\left|d_{12}\right|^{2}} \rho^{1} \rho^{2}\left[\left(U^{2}\right)^{\dagger} U^{1}-\left(U^{1}\right)^{\dagger} U^{2}\right]+\frac{\rho^{1} \rho^{2}\left(\rho^{3}\right)^{2} d_{12}^{M} \sigma_{M}}{4\left|d_{12}\right|^{2}\left|d_{13}\right|^{2}\left|d_{23}\right|^{2}} \\
& \times\left\{\left[\left(U^{3}\right)^{\dagger} U^{2}-\left(U^{2}\right)^{\dagger} U^{3}\right] d_{23}^{\dagger} d_{31}\left[\left(U^{1}\right)^{\dagger} U^{3}-\left(U^{3}\right)^{\dagger} U^{1}\right]\right. \\
& \left.-\left[\left(U^{3}\right)^{\dagger} U^{1}-\left(U^{1}\right)^{\dagger} U^{3}\right] d_{31}^{\dagger} d_{32}\left[\left(U^{2}\right)^{\dagger} U^{3}-\left(U^{3}\right)^{\dagger} U^{2}\right]\right\}+\mathcal{O}\left(d^{-5}\right), \\
r_{4}^{M} \sigma_{M} & =\frac{d_{13}^{M} \sigma_{M}}{\left|d_{13}\right|^{2}} \rho^{1} \rho^{3}\left[\left(U^{3}\right)^{\dagger} U^{1}-\left(U^{1}\right)^{\dagger} U^{3}\right]+\frac{\rho^{1} \rho^{3}\left(\rho^{2}\right)^{2} d_{13}^{M} \sigma_{M}}{4\left|d_{12}\right|^{2}\left|d_{13}\right|^{2}\left|d_{23}\right|^{2}} \\
& \times\left\{\left[\left(U^{2}\right)^{\dagger} U^{3}-\left(U^{3}\right)^{\dagger} U^{2}\right] d_{23}^{\dagger} d_{21}\left[\left(U^{1}\right)^{\dagger} U^{2}-\left(U^{2}\right)^{\dagger} U^{1}\right]\right. \\
& \left.-\left[\left(U^{2}\right)^{\dagger} U^{1}-\left(U^{1}\right)^{\dagger} U^{2}\right] d_{21}^{\dagger} d_{23}\left[\left(U^{3}\right)^{\dagger} U^{2}-\left(U^{2}\right)^{\dagger} U^{3}\right]\right\}+\mathcal{O}\left(d^{-5}\right), \\
r_{6}^{M} \sigma_{M} & =\frac{d_{23}^{M} \sigma_{M}}{\left|d_{23}\right|^{2}} \rho^{2} \rho^{3}\left[\left(U^{3}\right)^{\dagger} U^{2}-\left(U^{2}\right)^{\dagger} U^{3}\right]+\frac{\rho^{2} \rho^{3}\left(\rho^{1}\right)^{2} d_{23}^{M} \sigma_{M}}{4\left|d_{12}\right|^{2}\left|d_{13}\right|^{2}\left|d_{23}\right|^{2}} \\
& \times\left\{\left[\left(U^{1}\right)^{\dagger} U^{3}-\left(U^{3}\right)^{\dagger} U^{1}\right] d_{13}^{\dagger} d_{12}\left[\left(U^{2}\right)^{\dagger} U^{1}-\left(U^{1}\right)^{\dagger} U^{2}\right]\right. \\
& \left.-\left[\left(U^{1}\right)^{\dagger} U^{2}-\left(U^{2}\right)^{\dagger} U^{1}\right] d_{12}^{\dagger} d_{13}\left[\left(U^{3}\right)^{\dagger} U^{1}-\left(U^{1}\right)^{\dagger} U^{3}\right]\right\}+\mathcal{O}\left(d^{-5}\right),
\end{aligned}
$$

with the definition of the distance vector $d_{i j}$ between the $i$-th and $j$-th baryon,

$$
d_{i j}=d_{i j}^{M} \sigma_{M} .
$$

According to (3.16), we have the positions for the three baryons respectively,

$$
r^{M}=r_{3}^{M} / 2+r_{8}^{M} / 2 \sqrt{3},-r_{3}^{M} / 2+r_{8}^{M} / 2 \sqrt{3},-r_{8}^{M} / \sqrt{3},
$$

with

$$
\begin{aligned}
& d_{12}=-d_{21}=r_{3} \\
& d_{13}=-d_{31}=\left(r_{3}-\sqrt{3} r_{8}\right) / 2, \\
& d_{23}=-d_{32}=-\left(r_{3}+\sqrt{3} r_{8}\right) / 2 .
\end{aligned}
$$

\section{B. Substitute the ADHM data to the Lagrangian}

Here we use the $S U(2)$ matrices $U_{\dot{\alpha} i}^{A}$ to represent the rotation matrices for different three baryons with $A=1,2,3$, which can be written as $u_{0} \mathbf{1}_{2 \times 2}+i \sum_{i=1}^{3} u_{i} \tau^{i}$ with $\sum_{i=1}^{3}\left(u_{i}\right)^{2}=1$. Thus the terms consists of $U$ 's such as in (3.18) can be written explicitly as

$$
U_{\dot{\alpha} i}^{A}\left(U_{\dot{\beta} i}^{B}\right)^{\dagger}=u_{0}^{(A B)}\left(\mathbf{1}_{2 \times 2}\right)_{\dot{\alpha} \dot{\beta}}+i \sum_{i=1}^{3} u_{i}^{(A B)} \tau_{\dot{\alpha} \dot{\beta}}^{i} .
$$


where $u_{0}$ is defined as same as in (2.6). So we have new terms with new parameters $r_{a}^{M}$ with $a=1,4,6$ and $u_{0}^{A B}$ if compared with the ADHM data in (3.1).

As the case in the previous section, we also need to write the terms including $A_{0}$, which are the kinetic terms of $X$ and $\omega$ plus the CS term. For the kinetic term of $X$, we have,

$$
\operatorname{Tr}\left(D_{0} X^{M}\right)^{2}=\operatorname{Tr}\left(-i\left[A_{0}, \sum_{a=1}^{8} \frac{\lambda^{a}}{2} r_{a}^{M}\right]\right)^{2} .
$$

And (3.23) could be simplified as

$$
\begin{aligned}
\operatorname{Tr}\left(D_{0} X^{M}\right)^{2}= & \frac{1}{8}\left\{\left(A_{0}^{4}\right)^{2} r_{1}^{2}+\left(A_{0}^{6}\right)^{2} r_{1}^{2}+4\left(A_{0}^{1}\right)^{2} r_{3}^{2}+\left(A_{0}^{4}\right)^{2} r_{3}^{2}+\left(A_{0}^{6}\right)^{2} r_{3}^{2}\right. \\
& -2 A_{0}^{1} A_{0}^{4} r_{1} r_{4}-2 \sqrt{3} A_{0}^{6} A_{0}^{8} r_{1} r_{4}+6 A_{0}^{1} A_{0}^{6} r_{3} r_{4}-2 \sqrt{3} A_{0}^{4} A_{0}^{8} r_{3} r_{4} \\
& +\left(A_{0}^{1}\right)^{2} r_{4}^{2}+\left(A_{0}^{6}\right)^{2} r_{4}^{2}+3\left(A_{0}^{8}\right)^{2} r_{4}^{2}-2 A_{0}^{1} A_{0}^{6} r_{1} r_{6}-2 \sqrt{3} A_{0}^{4} A_{0}^{8} r_{1} r_{6} \\
& -6 A_{0}^{1} A_{0}^{4} r_{3} r_{6}+2 \sqrt{3} A_{0}^{6} A_{0}^{8} r_{3} r_{6}-2 A_{0}^{4} A_{0}^{6} r_{4} r_{6}+4 \sqrt{3} A_{0}^{1} A_{0}^{8} r_{4} r_{6} \\
& +\left(A_{0}^{1}\right)^{2} r_{6}^{2}+\left(A_{0}^{4}\right)^{2} r_{6}^{2}+3\left(A_{0}^{8}\right)^{2} r_{6}^{2}+\left(A_{0}^{3}\right)^{2}\left[4 r_{1}^{2}+r_{4}^{2}+r_{6}^{2}\right] \\
& +4 \sqrt{3} A_{0}^{4} A_{0}^{6} r_{1} r_{8}+2 \sqrt{3}\left(A_{0}^{4}\right)^{2} r_{3} r_{8}-2 \sqrt{3}\left(A_{0}^{6}\right)^{2} r_{3} r_{8}-2 \sqrt{3} A_{0}^{1} A_{0}^{6} r_{4} r_{8} \\
& -6 A_{0}^{4} A_{0}^{8} r_{4} r_{8}-2 \sqrt{3} A_{0}^{1} A_{0}^{4} r_{6} r_{8}-6 A_{0}^{6} A_{0}^{8} r_{6} r_{8}+3\left(A_{0}^{4}\right)^{2} r_{8}^{2}+3\left(A_{0}^{6}\right)^{2} r_{8}^{2} \\
& -2 A_{0}^{3}\left[4 A_{0}^{1} r_{1} r_{3}+3 A_{0}^{6} r_{1} r_{4}+A_{0}^{4} r_{3} r_{4}-\sqrt{3} A_{0}^{8} r_{4}^{2}-3 A_{0}^{4} r_{1} r_{6}+A_{0}^{6} r_{3} r_{6}\right. \\
& \left.\left.+\sqrt{3} A_{0}^{8} r_{6}^{2}+\sqrt{3} A_{0}^{4} r_{4} r_{8}-\sqrt{3} A_{0}^{6} r_{6} r_{8}\right]\right\} .
\end{aligned}
$$

Note that (3.24) does not include the terms of $A_{0}^{a}$ with $a=2,5,7$. We have omitted these terms since all $A_{0}^{a}$ with $a=2,5,7$ appear in the Lagrangian as quadratic terms which yields $A_{0}^{a=2,5,7}=0$ by their equations of motion. According to these, we have the kinetic term for $\omega$ which is similar to the case of the 't Hooft instanton as follow, 


$$
\begin{aligned}
\operatorname{Tr}\left(D_{0} \bar{\omega}_{i}^{\dot{\alpha}} D_{0} \omega_{\dot{\alpha} i}\right)= & 2\left[\left(\rho^{1}\right)^{2}+\left(\rho^{2}\right)^{2}+\left(\rho^{3}\right)^{2}\right]\left[\left(A_{0}^{0}\right)^{2}+\frac{1}{6} \sum_{a=1,3,4,6,8}\left(A_{0}^{a}\right)^{2}\right]+4 \rho^{1} \rho^{2} u_{0}^{(12)} A_{0}^{1} A_{0}^{0} \\
& +4 \rho^{1} \rho^{3} u_{0}^{(13)} A_{0}^{4} A_{0}^{0}+4 \rho^{2} \rho^{3} u_{0}^{(23)} A_{0}^{6} A_{0}^{0}+2 A_{0}^{3} A_{0}^{0}\left[\left(\rho^{1}\right)^{2}-\left(\rho^{2}\right)^{2}\right] \\
& +\frac{2}{\sqrt{3}} A_{0}^{8} A_{0}^{0}\left[\left(\rho^{1}\right)^{2}+\left(\rho^{2}\right)^{2}-2\left(\rho^{3}\right)^{2}\right]+\frac{2 \rho^{1} \rho^{2} u_{0}^{(12)}}{\sqrt{3}} A_{0}^{1} A_{0}^{8}+\rho^{1} \rho^{2} u_{0}^{(12)} A_{0}^{4} A_{0}^{6} \\
& -\frac{\rho^{1} \rho^{2} u_{0}^{(13)}}{\sqrt{3}} A_{0}^{4} A_{0}^{8}+\rho^{1} \rho^{3} u_{0}^{(13)} A_{0}^{1} A_{0}^{6}+\rho^{1} \rho^{3} u_{0}^{(13)} A_{0}^{3} A_{0}^{4} \\
& -\frac{\rho^{2} \rho^{3} u_{0}^{(23)}}{\sqrt{3}} A_{0}^{6} A_{0}^{8}+\rho^{2} \rho^{3} u_{0}^{(23)} A_{0}^{1} A_{0}^{4}-\rho^{2} \rho^{3} u_{0}^{(23)} A_{0}^{3} A_{0}^{6} \\
& +\left[\frac{1}{\sqrt{3}} A_{0}^{3} A_{0}^{8}+\frac{1}{4}\left(A_{0}^{4}\right)^{2}-\frac{1}{4}\left(A_{0}^{6}\right)^{2}\right]\left[\left(\rho^{1}\right)^{2}-\left(\rho^{2}\right)^{2}\right] \\
& +\frac{1}{12}\left[2\left(A_{0}^{1}\right)^{2}+2\left(A_{0}^{3}\right)^{2}-2\left(A_{0}^{8}\right)^{2}-\left(A_{0}^{4}\right)^{2}-\left(A_{0}^{6}\right)^{2}\right]\left[\left(\rho^{1}\right)^{2}+\left(\rho^{2}\right)^{2}-2\left(\rho^{3}\right)^{2}\right] .
\end{aligned}
$$

We have used $\omega_{\dot{\alpha} i}^{A} \lambda_{A B}^{a}\left(\omega_{\dot{\alpha} i}^{B}\right)^{*}=0$ for $a=2,5,7$ since they are proportional to $U_{\dot{\alpha} i}^{A}\left(U_{\dot{\alpha} i}^{B}\right)^{\dagger}-U_{\dot{\alpha} i}^{B}\left(U_{\dot{\alpha} i}^{A}\right)^{\dagger}$ with $A, B=1,2,3$. So the total Lagrangian can be written as the form in (3.6) again with the CS term given in (3.5).

Additionally, we have another term to the "on-shell" Lagrangian which comes from the mass term of $X^{4}$ in this D0-D4 matrix model,

$$
\begin{aligned}
\frac{\lambda N_{c} M_{K K}}{54 \pi}(1+\zeta)^{3 / 2} \frac{2}{3}\left(1-\frac{1}{2} \zeta\right) M_{K K}^{2} \operatorname{Tr}\left(X^{4}\right)^{2}= & \frac{\lambda N_{c} M_{K K}^{3}}{3^{4} \pi}\left(1-\frac{1}{2} \zeta\right)(1+\zeta)^{3 / 2}\left[\frac{1}{4}\left(r_{3}^{4}+\frac{1}{\sqrt{3}} r_{8}^{4}\right)^{2}\right. \\
& \left.+\frac{1}{4}\left(-r_{3}^{4}+\frac{1}{\sqrt{3}} r_{8}^{4}\right)^{2}+\frac{1}{3}\left(r_{8}^{4}\right)^{2}+\frac{1}{2} \sum_{\rho=1,2,4,5,6,7}\left(r_{\rho}^{4}\right)^{2}\right]
\end{aligned}
$$

The two- and three-body terms are in the last term of (3.26) while the first three terms are related to one baryon potential. So we need to write the expressions for the off-diagonal $r_{1,2,4,5,6,7}$ to evaluate them.

Basically, the three-body force could be determined in principle by straightforward calculations from (3.24) (3.25) (3.26) and (3.5). However the calculations would be very messy and we are less clear about how to obtain a physical interpretation from the calculations. To clarify the physical essence, we therefore are going to employ the arguments as in [19] i.e. choose a particular alignment of the baryons. And the physical essence and significance would be clear by this choice.

\subsection{Hamiltonian for three baryons aligned on a line}

We consider the following condition as in [19] for the baryons aligned on a line,

$$
r_{8}^{M}=0, \quad r_{3}^{M} \equiv r^{M} \neq 0
$$


which means three baryons are located at $x^{M}=r_{3}^{M} / 2, x^{M}=-r_{3}^{M} / 2$ and $x^{M}=0$ respectively. The resultant Lagrangian would be simplified as

$$
L_{A_{0}}=\frac{\lambda N_{c} M_{K K}}{54 \pi}(1+\zeta)^{3 / 2}\left(L_{1}+L_{2}\right)
$$

where

$$
\begin{aligned}
L_{1}= & \frac{162 \pi A_{0}^{0}}{\lambda M_{K K}(1+\zeta)^{3 / 2}}+\frac{\left(A_{0}^{1}\right)^{2} r^{2}}{2}+\frac{\left(A_{0}^{4}\right)^{2} r^{2}}{8}+\frac{\left(A_{0}^{6}\right)^{2} r^{2}}{8} \\
& +\left[6\left(A_{0}^{0}\right)^{2}+\left(A_{0}^{1}\right)^{2}+\left(A_{0}^{3}\right)^{2}+\left(A_{0}^{4}\right)^{2}+\left(A_{0}^{6}\right)^{2}+\left(A_{0}^{8}\right)^{2}\right] \rho^{2} \\
& +\left(A_{0}^{4} A_{0}^{6}+\frac{2 A_{0}^{1} A_{0}^{8}}{\sqrt{3}}\right) \rho^{2} u_{0}^{12}+\left(A_{0}^{3} A_{0}^{4}+A_{0}^{1} A_{0}^{6}-\frac{A_{0}^{4} A_{0}^{8}}{\sqrt{3}}\right) \rho^{2} u_{0}^{(13)} \\
& +\left(A_{0}^{1} A_{0}^{4}-A_{0}^{3} A_{0}^{6}-\frac{A_{0}^{6} A_{0}^{8}}{\sqrt{3}}\right) \rho^{2} u_{0}^{(23)}+4 A_{0}^{0}\left(A_{0}^{1} u_{0}^{(12)}+A_{0}^{4} u_{0}^{(13)}+A_{0}^{6} u_{0}^{(23)}\right) \rho^{2}, \\
L_{2}= & \frac{1}{4}\left[2\left(A_{0}^{3}\right)^{2} r_{1}^{2}+\frac{1}{2}\left(A_{0}^{4}\right)^{2} r_{1}^{2}+\frac{1}{2}\left(A_{0}^{6}\right)^{2} r_{1}^{2}-A_{0}^{1} A_{0}^{4} r_{1} r_{4}-3 A_{0}^{3} A_{0}^{6} r_{1} r_{4}\right. \\
& -\sqrt{3} A_{0}^{6} A_{0}^{8} r_{1} r_{4}+\frac{1}{2}\left(A_{0}^{1}\right)^{2} r_{4}^{2}+\frac{1}{2}\left(A_{0}^{3}\right)^{2} r_{4}^{2}+\frac{1}{2}\left(A_{0}^{6}\right)^{2} r_{4}^{2}+\sqrt{3} A_{0}^{3} A_{0}^{8} r_{4}^{2} \\
& +\frac{3}{2}\left(A_{0}^{8}\right)^{2} r_{4}^{2}+3 A_{0}^{3} A_{0}^{4} r_{1} r_{6}-A_{0}^{1} A_{0}^{6} r_{1} r_{6}-\sqrt{3} A_{0}^{4} A_{0}^{8} r_{1} r_{6}-A_{0}^{4} A_{0}^{6} r_{4} r_{6} \\
& +2 \sqrt{3} A_{0}^{1} A_{0}^{8} r_{4} r_{6}+\frac{1}{2}\left(A_{0}^{1}\right)^{2} r_{6}^{2}+\frac{1}{2}\left(A_{0}^{3}\right)^{2} r_{6}^{2}+\frac{1}{2}\left(A_{0}^{4}\right)^{2} r_{6}^{2} \\
& \left.-\sqrt{3} A_{0}^{3} A_{0}^{8} r_{6}^{2}+\frac{3}{2}\left(A_{0}^{8}\right)^{2} r_{6}^{2}\right] .
\end{aligned}
$$

For obtaining the expression (3.29), the terms related to $r_{3,8}$ and $Y$ have been eliminated since we have used the following equations

$$
r_{3}^{M} r_{1}^{M}=0,\left(r_{3}^{M}+\sqrt{3} r_{8}^{M}\right) r_{4}^{M}=0,\left(r_{3}^{M}-\sqrt{3} r_{8}^{M}\right) r_{6}^{M}=0 .
$$

which could be explicitly shown by the ADHM constraint (2.2) in the expansion of $|r| \gg \rho$ (See the details of this expansion in [23]). Then we have to evaluate the mass term of $X^{4}$ in this matrix model, as an explicit result it is

$$
\begin{aligned}
V_{3-\text { body }}^{\text {mass }}= & \frac{\lambda N_{c} M_{K K}^{3}}{2^{2} 3^{4} \pi}\left(1-\frac{1}{2} \zeta\right)(1+\zeta)^{3 / 2} \frac{\rho^{6}}{|r|^{6}} \\
& \times\left\{\operatorname{Tr}\left(r T_{12}\right) \operatorname{Tr}\left[r\left(T_{23} T_{13}-T_{13} T_{23}\right)\right]-2 \operatorname{Tr}\left(r T_{31}\right) \operatorname{Tr}\left[r\left(T_{32} T_{12}-T_{12} T_{32}\right)\right]\right. \\
& \left.-2 \operatorname{Tr}\left(r T_{32}\right) \operatorname{Tr}\left[r\left(T_{31} T_{21}-T_{21} T_{31}\right)\right]\right\},
\end{aligned}
$$

where $r=r^{M} \sigma_{M}$ and $T_{i j}=\left(U^{i}\right)^{\dagger} U^{j}-\left(U^{j}\right)^{\dagger} U^{i}=-T_{j i}$. And we have used (3.30) to simplify (3.18) for these aligned baryons to obtain (3.31) as 


$$
\begin{aligned}
& r_{1}^{M} \sigma_{M}=\frac{1}{|r|^{2}} \rho^{1} \rho^{2} r T_{21}-\frac{1}{|r|^{4}} \rho^{1} \rho^{2}\left(\rho^{3}\right)^{2} r\left(T_{32} T_{13}-T_{13} T_{32}\right)+\mathcal{O}\left(1 /|r|^{5}\right), \\
& r_{4}^{M} \sigma_{M}=\frac{2}{|r|^{2}} \rho^{1} \rho^{3} r T_{31}-\frac{1}{|r|^{4}} \rho^{1} \rho^{3}\left(\rho^{2}\right)^{2} r\left(T_{32} T_{12}-T_{12} T_{32}\right)+\mathcal{O}\left(1 /|r|^{5}\right), \\
& r_{6}^{M} \sigma_{M}=\frac{1}{|r|^{2}} \rho^{2} \rho^{3} r T_{32}-\frac{1}{|r|^{4}} \rho^{2} \rho^{3}\left(\rho^{1}\right)^{2} r\left(T_{31} T_{21}-T_{21} T_{31}\right)+\mathcal{O}\left(1 /|r|^{5}\right) .
\end{aligned}
$$

Note that only the second terms in each right hand side of (3.32) are related to the three-body case while the first terms in the right hand side of (3.32) equal to the off-diagonal entry of two-body case. This has been considered in the potential of (3.31) with taking the classical value as $\rho^{1}=\rho^{2}=\rho^{3}=\rho$ for the leading term in the large $N_{c}$ expansion.

\section{Integrate out the auxiliary field $A_{0}$}

We also need to solve the equations of motion for $A_{0}$ derived from Lagrangian (3.29). By plugging the solution back into (3.29), we obtain

$$
L_{A_{0}}=-V, \quad V=\sum_{A=1,2,3} V_{1-\text { body }}^{(A)}+\frac{1}{2} \sum_{A \neq B} V_{2-\text { body }}^{(A, B)}+V_{3-\text { body }} .
$$

As (2.6) or in [8], the expressions for one- and two-body potential are

$$
V_{1-\text { body }}^{(A)}=\frac{27 \pi N_{c}}{4 \lambda M_{K K}(1+\zeta)^{3 / 2}} \frac{1}{\rho^{2}}, \quad V_{2-\text { body }}^{(A, B)}=\frac{27 \pi N_{c}}{\lambda M_{K K}(1+\zeta)^{3 / 2}} \frac{\left(u_{0}^{(A B)}\right)^{2}}{\left|r^{(A B)}\right|^{2}+2 \rho^{2}-2\left(u_{0}^{(A B)}\right)^{2} \rho^{2}} .
$$

By the condition (3.27) for the aligned baryons

$$
\left|r^{(12)}\right|=r, \quad\left|r^{(13)}\right|=\left|r^{(23)}\right|=r / 2,
$$

and then we will compute the three-body potential $V_{3 \text {-body }}$ in (3.33).

As we are going to use the same trick as in [19] to solve the equations of motion for $A_{0}$, we first rewrite the Lagrangian $L_{1}$ as

$$
L_{1}=A^{T} M A+B^{T} A
$$

where

$$
A^{T}=\left(A_{0}^{0}, A_{0}^{1}, A_{0}^{3}, A_{0}^{4}, A_{0}^{6}, A_{0}^{8}\right), \quad B^{T}=\frac{162 \pi}{\lambda M_{K K}(1+\zeta)^{3 / 2}}(1,0,0,0,0,0)
$$

and 


$$
M=\rho^{2}\left(\begin{array}{cccccc}
6 & 2 u_{0}^{(12)} & 0 & 2 u_{0}^{(13)} & 2 u_{0}^{(23)} & 0 \\
2 u_{0}^{(12)} & 1+r^{2} / 2 \rho^{2} & 0 & u_{0}^{(23)} / 2 & u_{0}^{(13)} / 2 & u_{0}^{(12)} / \sqrt{3} \\
0 & 0 & 1 & u_{0}^{(13)} / 2 & -u_{0}^{(23)} / 2 & 0 \\
2 u_{0}^{(13)} & u_{0}^{(23)} / 2 & u_{0}^{(13)} / 2 & 1+r^{2} / 8 \rho^{2} & u_{0}^{(12)} / 2 & -u_{0}^{(13)} / 2 \sqrt{3} \\
2 u_{0}^{(23)} & u_{0}^{(13)} / 2 & -u_{0}^{(23)} / 2 & u_{0}^{(12)} / 2 & 1+r^{2} / 8 \rho^{2} & -u_{0}^{(23)} / 2 \sqrt{3} \\
0 & u_{0}^{(12)} / \sqrt{3} & 0 & -u_{0}^{(13)} / 2 \sqrt{3} & -u_{0}^{(23)} / 2 \sqrt{3} & 1
\end{array}\right)
$$

There should be another Lagrangian $L_{2}$ for the computation, however it turns out that Lagrangian $L_{2}$ is not necessary in the next computation since our computation is in a "long-distance" expansion $\rho \ll r$ and Lagrangian $L_{2}$ is at higher order in this expansion 7 .

By (3.36), the solution for the equation of motion for $A_{0}$ is

$$
A=-\frac{1}{2} M^{-1} B
$$

and the Hamiltonian is therefore

$$
\begin{aligned}
V & =\frac{\lambda M_{K K} N_{c}}{54 \pi}(1+\zeta)^{3 / 2} \frac{1}{4} B^{T} M^{-1} B \\
& =\frac{3^{5} \pi N_{c}}{2 \lambda M_{K K}(1+\zeta)^{3 / 2}}\left[M^{-1}\right]_{(1,1)} .
\end{aligned}
$$

We obtain the following leading term by expanding in power series of $\rho^{2} / r^{2}$

$$
V=\frac{3^{5} \pi N_{c}}{2 \lambda M_{K K}(1+\zeta)^{3 / 2}}\left[\frac{1}{6 \rho^{2}}+\frac{2\left(u^{(1,2)}\right)^{2}+8\left(u^{(1,3)}\right)^{2}+8\left(u^{(2,2)}\right)^{2}}{9 r^{2}}+\frac{4 \rho^{2} f_{S I}}{9 r^{4}}\right]+\mathcal{O}\left(\rho^{4} / r^{6}\right),
$$

where the function $f_{S I}$ is the spin/isospin phase defined as

$$
\begin{aligned}
f_{S I} & =\left(u_{0}^{(1,2)}\right)^{4}-\left(u_{0}^{(1,2)}\right)^{2}+16\left(u_{0}^{(1,3)}\right)^{4}-16\left(u_{0}^{(1,3)}\right)^{2}+16\left(u_{0}^{(1,3)}\right)^{4}+16\left(u_{0}^{(2,3)}\right)^{4}-16\left(u_{0}^{(2,3)}\right)^{2} \\
& +4\left(u_{0}^{(1,2)}\right)^{2}\left(u_{0}^{(1,3)}\right)^{2}+4\left(u_{0}^{(1,2)}\right)^{2}\left(u_{0}^{(2,2)}\right)^{2}+16\left(u_{0}^{(1,3)}\right)^{2}\left(u_{0}^{(2,3)}\right)^{2}-24 u_{0}^{(1,2)} u_{0}^{(2,3)} u_{0}^{(1,3)} .
\end{aligned}
$$

Subtracting the one- and two-body potential (3.34) from (3.41), we obtain the three-body potential in the expansion of $\rho^{2} / r^{2}$ as

$$
\begin{aligned}
V_{3-\text { body }}^{A_{0}} & =\frac{216 \pi N_{c} \rho^{2}}{\lambda M_{K K}(1+\zeta)^{3 / 2}|r|^{4}}\left[\left(u_{0}^{(1,2)}\right)^{2}\left(u_{0}^{(1,3)}\right)^{2}+\left(u_{0}^{(1,2)}\right)^{2}\left(u_{0}^{(2,3)}\right)^{2}+4\left(u_{0}^{(1,3)}\right)^{2}\left(u_{0}^{(2,3)}\right)^{2}\right. \\
& \left.-6 u_{0}^{(1,2)} u_{0}^{(2,3)} u_{0}^{(1,3)}\right]+\mathcal{O}\left(\rho^{4} / r^{6}\right) .
\end{aligned}
$$

\footnotetext{
${ }^{7} \mathrm{As}$ in [19, we have also checked this to confirm that Lagrangian $L_{2}$ is indeed at higher order for the next computation. However the computation is lengthy and not necessary for this manuscript thus it is not presented here.
} 
With the mass term for $X^{4}$ (3.31), we have the total three-body potential which is

$$
V_{3-\text { body }}=V_{3-\text { body }}^{A_{0}}+V_{3-\text { body }}^{\text {mass }} .
$$

We can evaluate the potential with a three-body baryonic state with (3.43) and (3.44) for any spin or isospin. So we will choose two different baryonic states as in [19] to study the three-body nuclear potential.

Furthermore, we also have some comments about (3.43). As mentioned that $\rho$ is of order $\mathcal{O}(1 / \sqrt{\lambda})$, thus the three-body Hamiltonian is of order $\mathcal{O}\left(1 / \lambda^{2} r^{4}\right)$ which is therefore suppressed by $1 / \lambda^{2}$. It is also consistent with [20] in which the generic $k$-body potential is of order $\mathcal{O}\left(1 / \lambda^{k-1} r^{2 k-2}\right)$ with $k=3$ and $M_{K K}=1$ if setting $\zeta=0$, i.e. no smeared D0-branes. Additionally, if all the matrices $U^{(i)}$ 's in (3.44) equal to each other which means the ADHM data returns to the 't Hooft instantons, we would have $u_{0}^{(i, j)}=1$ and $A_{i j}=0$, yielding the vanishing three-body potential as same as in (3.14) in this D0-D4/D8 system. Thus obviously it is a consistent check for the results in the previous section.

\section{Evaluate the potential with baryonic states}

In this subsection, we are going to compute the spin/isospin dependence of the three-body short-distance force with our three-body potential from the D0-D4/D8 matrix model. As a parallel study, we would like to choose the following two states as in [19] which are

1. three-neutrons with averaged spins.

2. proton-proton-neutron (or proton-neutron-neutron).

The first state is relevant to the dense states of many neutrons as core of neutron stars or supernovae while the second state is for the spectrum of Helium-3 nucleus. In some high-density system, the non-trivial QCD vacuum may affect nuclear force among baryons, as a description, we would like to use our D0-D4/D8 matrix model to study the nuclear force with non-trivial QCD vacuum since the number density of D0-branes in this D0-D4/D8 system is, for example, relevant to the glueball condensation or CME [9, 10, 11].

(1) three-neutrons with averaged spins.

The single-baryon wave function has been given in (2.9) 8 for protons and neutrons. Since we need neutron states with averaged spins, thus for any given operators, the appropriate expectation is

$$
\langle V\rangle=\frac{1}{2}[\langle n \uparrow|\mathcal{O}| n \uparrow\rangle+\langle n \downarrow|\mathcal{O}| n \downarrow\rangle] .
$$

We need to take the expectation value for three baryons for $\mathcal{O}$ being the three-body Hamiltonian. Here we will not anti-symmetrize the wave function although the nucleons are fermions. We consider a single baryon case as (3.45), it yields

$$
\langle V\rangle=\int d \Omega_{3} \frac{1}{2}\left[\mathcal{O}|\langle\vec{a} \mid n \uparrow\rangle|^{2}+\mathcal{O}|\langle\vec{a} \mid n \downarrow\rangle|^{2}\right] .
$$

The $d \Omega_{3}$ is the integration over $S^{3}$ by the unit vector $\vec{a}$. By the wave function (2.9), we have

$$
|\langle\vec{a} \mid n \uparrow\rangle|^{2}+|\langle\vec{a} \mid n \downarrow\rangle|^{2}=\frac{1}{\pi^{2}}\left[\left(a_{1}\right)^{2}+\left(a_{2}\right)^{2}+\left(a_{3}\right)^{2}+\left(a_{4}\right)^{2}\right]=\frac{1}{\pi^{2}},
$$

\footnotetext{
${ }^{8}$ The wave function may be deformed if we consider the baryons or nucleon with non-zero QCD vacuum. Thus here we use (2.9) as an ansatz to study the nuclear force with non-zero QCD vacuum.
} 
therefore,

$$
\langle V\rangle=\frac{1}{2 \pi^{2}} \int d \Omega_{3} \mathcal{O} .
$$

So according to (3.48), with the spin-averaged wave function, the three-body potential would be,

$$
\begin{aligned}
\left\langle V_{3-\text { body }}^{A_{0}}\right\rangle_{\text {nnn(spin-averaged })}= & \frac{216 \pi N_{c} \rho^{2}}{\lambda M_{K K}(1+\zeta)^{3 / 2}|r|^{4}} \times \\
& \frac{1}{\left(2 \pi^{2}\right)^{3}} \int d \Omega_{3}^{(1)} d \Omega_{3}^{(2)} d \Omega_{3}^{(3)}\left[\left(u_{0}^{(1,2)}\right)^{2}\left(u_{0}^{(1,3)}\right)^{2}+\left(u_{0}^{(1,2)}\right)^{2}\left(u_{0}^{(2,3)}\right)^{2}\right. \\
& \left.4\left(u_{0}^{(1,3)}\right)^{2}\left(u_{0}^{(2,3)}\right)^{2}-6 u_{0}^{(1,2)} u_{0}^{(2,3)} u_{0}^{(1,3)}\right] .
\end{aligned}
$$

And the next computation is quite similar as done in [19]. For example, using $u_{0}=\frac{1}{2}\left(\operatorname{Tr}\left[\left(U^{1}\right)^{\dagger} U^{2}\right]\right)$ and $\left(a_{1}\right)^{2}+\left(a_{2}\right)^{2}+\left(a_{3}\right)^{2}+\left(a_{4}\right)^{2}=1$, thus for $\left(u_{0}^{(1,2)}\right)^{2}$ we have

$$
u_{0}^{(i, j)}=\frac{1}{2} \operatorname{Tr}\left[U^{(i) \dagger} U^{(j)}\right]=\vec{a}^{(i)} \cdot \vec{a}^{(j)},
$$

where $\vec{a}^{(i)}$ is unit 4-component vector, pointing one phase for spin or isospin on $S^{3}$ by the definition of $U$. Therefore, we can obtain

$$
\begin{aligned}
\int d \Omega_{3}^{(1)}\left(u_{0}^{(1,2)}\right)^{2}=\int d \Omega_{3}^{(1)} \cos ^{2} \theta=\int \cos ^{2} \theta \sin ^{2} \theta \sin \bar{\theta} d \theta d \bar{\theta} d \phi & =\frac{\pi^{2}}{2}, \\
\int d \Omega_{3}^{(1)} d \Omega_{3}^{(2)} d \Omega_{3}^{(3)} u_{0}^{(1,2)} u_{0}^{(2,3)} u_{0}^{(1,3)} & =\frac{\pi^{6}}{2},
\end{aligned}
$$

where $\theta$ is the angle between $\vec{a}^{(i)}$ and $\vec{a}^{(j)}$. Finally we obtain

$$
\left\langle V_{3-\text { body }}^{A_{0}}\right\rangle_{\text {nnn(spin-averaged) }}=0 .
$$

Therefore we also obtain a vanished three-body potential from the $A_{0}$ terms with the spin-averaged neutron wave function.

Similarly, we can obtain the expression of the expectation for $V_{3-\text { body }}^{\text {mass }}$ as 


$$
\begin{aligned}
\left\langle V_{3-\text { body }}^{\text {mass }}\right\rangle_{\text {nnn(spin-averaged })}= & \frac{\lambda N_{c} M_{K K}^{3}}{2^{2} 3^{4} \pi}\left(1-\frac{1}{2} \zeta\right)(1+\zeta)^{3 / 2} \frac{\rho^{6}}{|r|^{6}} \\
& \times \frac{1}{\left(2 \pi^{2}\right)^{3}} \int d \Omega_{3}^{(1)} d \Omega_{3}^{(2)} d \Omega_{3}^{(3)}\left\{\operatorname{Tr}\left(r T_{12}\right) \operatorname{Tr}\left[r\left(T_{23} T_{13}-T_{13} T_{23}\right)\right]\right. \\
& \left.-2 \operatorname{Tr}\left(r T_{31}\right) \operatorname{Tr}\left[r\left(T_{32} T_{12}-T_{12} T_{32}\right)\right]-2 \operatorname{Tr}\left(r T_{32}\right) \operatorname{Tr}\left[r\left(T_{31} T_{21}-T_{21} T_{31}\right)\right]\right\} \\
= & -\frac{\lambda N_{c} M_{K K}^{3}}{2^{2} 3^{3} \pi}\left(1-\frac{1}{2} \zeta\right)(1+\zeta)^{3 / 2} \frac{\rho^{6}}{|r|^{6}} \frac{1}{\left(2 \pi^{2}\right)^{3}} \\
& \times \int d \Omega_{3}^{(1)} d \Omega_{3}^{(2)} d \Omega_{3}^{(3)} \operatorname{Tr}\left(r T_{21}\right) \operatorname{Tr}\left[r\left(T_{23} T_{13}-T_{13} T_{23}\right)\right] .
\end{aligned}
$$

The integration of (3.53) could be performed by using the polar coordinates and we have used the symmetry for exchanging of the integration variables. As a result, we have the following integration

$$
\frac{1}{\left(2 \pi^{2}\right)^{3}} \int d \Omega_{3}^{(1)} d \Omega_{3}^{(2)} d \Omega_{3}^{(3)} \operatorname{Tr}\left(r T_{21}\right) \operatorname{Tr}\left[r\left(T_{23} T_{13}-T_{13} T_{23}\right)\right]=-8|\vec{r}|^{2} .
$$

Therefore we obtain the expectation from (3.53) which is

$$
\left\langle V_{3-\text { body }}^{\text {mass }}\right\rangle_{\operatorname{nnn}(\text { spin-averaged })}=\frac{2^{-1 / 2} 3^{15 / 2} \pi^{2} N_{c}}{\lambda^{2} M_{K K}^{3}\left(1-\frac{1}{2} \zeta\right)^{1 / 2}(1+\zeta)^{3}|\vec{r}|^{4}},
$$

where the three-dimensional vector $\vec{r}$ specifies the inter-baryon distance in our space. The four-dimensional distance could be identified as three-dimensional distance since we can choose the classical value for the four-dimensional component $r^{4}$ of $r^{M}=\left(\vec{r}, r^{4}\right)$ vanished at leading order in $1 / N$ expansion. And in (3.55), we have substituted the classical value of $\rho$, which is $\rho=2^{-1 / 4} 3^{7 / 4} \sqrt{\pi} \lambda^{-1 / 3} M_{K K}^{-1}\left(1-\frac{1}{2} \zeta\right)^{-1 / 4}(1+\zeta)^{-3 / 4}$ in [8], for two-flavor case also for the leading order in the $1 / N_{c}$ expansion. So we obtain the total three-body potential in our D0-D4/D8 system as,

$$
\begin{aligned}
\left\langle V_{3-\text { body }}\right\rangle_{\text {nnn(spin-averaged })} & =\left\langle V_{3-\text { body }}^{A_{0}}\right\rangle_{\text {nnn(spin-averaged })}+\left\langle V_{3-\text { body }}^{\text {mass }}\right\rangle_{\text {nnn(spin-averaged })} \\
& =\frac{2^{-1 / 2} 3^{15 / 2} \pi^{2} N_{c}}{\lambda^{2} M_{K K}^{3}\left(1-\frac{1}{2} \zeta\right)^{1 / 2}(1+\zeta)^{3}|\vec{r}|^{4}} .
\end{aligned}
$$

This three-body force is obtained by considering effect of non-trivial QCD vacuum in the D0-D4/D8 system and also with averaged spin. The three-body force is suppressed if compared to the two-body potential (2.10) for large $\lambda$. As in [20], our three-body potential is also a generic hierarchy between $N+1$ - to $N$-body potential. $M_{K K}$ is the energy scale for the dual QCD-like field theory, and our calculations are as well valid at short distances. However if we focus on the factor $\left(1-\frac{1}{2} \zeta\right)^{1 / 2}$, it implies that the three-body potential is totally complex with $\zeta>2$, which is nothing but our constraint for stable baryonic state in this system. We will discuss about it in details in the final section.

(2) proton-proton-neutron (or proton-neutron-proton). 
In this subsection, let us evaluate the three-body potential with the state of proton-proton-neutron (which is also a same calculation for the case of proton-neutron-proton). We will consider the three-nucleon state with a total spin $1 / 2$ and a total isospin $1 / 2$. We can use the following state to represent a proton-proton-neutron state with the third component of the total isospin $+1 / 2$,

$$
\begin{aligned}
& \frac{1}{\sqrt{6}}\left[\left|p \uparrow>_{1}\right| p \downarrow>_{2}\left|n \downarrow>_{3}-\right| p \downarrow>_{1}\left|p \uparrow>_{2}\right| n \uparrow>_{3}-\left|p \uparrow>_{1} n \uparrow>_{2}\right| p \downarrow>_{3}\right. \\
& \left.\quad+\left|p \downarrow>_{1}\right| n \uparrow>_{2}\left|p \uparrow>_{3}-\right| n \uparrow>_{1}\left|p \downarrow>_{2}\right| p \uparrow>_{3}+\left|n \uparrow>_{1}\right| p \uparrow>_{2} \mid p \downarrow>_{3}\right] .
\end{aligned}
$$

The next calculations are straightforward and similar to what we have done for three-neutrons with averaged spins. With the following integrals

$$
\begin{aligned}
\int d \Omega_{3}^{(1)} d \Omega_{3}^{(2)} d \Omega_{3}^{(3)}\left|\psi\left(\vec{a}_{1}, \vec{a}_{2}, \vec{a}_{3}\right)\right|^{2}\left(u_{0}^{(1,2)}\right)^{2}\left(u_{0}^{(1,3)}\right)^{2} & =\frac{1}{36}, \\
\int d \Omega_{3}^{(1)} d \Omega_{3}^{(2)} d \Omega_{3}^{(3)}\left|\psi\left(\vec{a}_{1}, \vec{a}_{2}, \vec{a}_{3}\right)\right|^{2} u_{0}^{(1,2)} u_{0}^{(2,3)} u_{0}^{(1,3)} & =\frac{1}{36}, \\
\int d \Omega_{3}^{(1)} d \Omega_{3}^{(2)} d \Omega_{3}^{(3)}\left|\psi\left(\vec{a}_{1}, \vec{a}_{2}, \vec{a}_{3}\right)\right|^{2} \operatorname{Tr}\left(r T_{21}\right) \operatorname{Tr}\left[r\left(T_{23} T_{13}-T_{13} T_{23}\right)\right] & =-\frac{320}{27}|\vec{r}|^{2} .
\end{aligned}
$$

With these formulas, we have

$$
\begin{aligned}
\left\langle V_{3-\text { body }}^{A_{0}}\right\rangle_{\mathrm{ppn}} & =0 \\
\left\langle V_{3-\text { body }}^{\text {mass }}\right\rangle_{\mathrm{ppn}} & =\frac{2^{5 / 2} 3^{9 / 2} 5 \pi^{2} N_{c}}{\lambda^{2} M_{K K}^{3}\left(1-\frac{1}{2} \zeta\right)^{1 / 2}(1+\zeta)^{3}|\vec{r}|^{4}} .
\end{aligned}
$$

Therefore we have the total three-body potential for proton-proton-neutron which is

$$
\begin{aligned}
\left\langle V_{3-\text { body }}\right\rangle_{\mathrm{ppn}} & =\left\langle V_{3-\text { body }}^{A_{0}}\right\rangle_{\mathrm{ppn}}+\left\langle V_{3-\text { body }}^{\text {mass }}\right\rangle_{\mathrm{ppn}} \\
& =\frac{2^{5 / 2} 3^{9 / 2} 5 \pi^{2} N_{c}}{\lambda^{2} M_{K K}^{3}\left(1-\frac{1}{2} \zeta\right)^{1 / 2}(1+\zeta)^{3}|\vec{r}|^{4}} .
\end{aligned}
$$

This three-body potential is positive which means there is a repulsive three-body force at short distances. The computation for three other wave functions $(+1 / 2,-1 / 2),(-1 / 2,+1 / 2)$ and $(-1 / 2,-1 / 2)$ of the third component of the spin and isospin, is the same as the current computation for $(+1 / 2,+1 / 2)$. And the result turns out to be the same as (3.60) since our matrix model (2.1) is $S O(3)$ invariance for rotational symmetry and $S U(2)$ invariance for isospin symmetry.

\section{Summary and discussion}

We proposed a matrix model with $U(k)$ gauge symmetry in [8] for $k$-body baryon systems with non-trivial QCD vacuum. And in this paper, we use this matrix model to compute the three-body force for baryons at 
short distances. We find the result includes some effects, maybe such as in glueball condensation and CME, from the non-trivial QCD vacuum. We derived the matrix model by using the standard technique from gauge/string duality (also the AdS/CFT correspondence), thus our matrix model is not a phenomenological model. Precisely, the matrix model is a low-energy effective theory for the baryon vertex, which is denoted as a D4'-brane, in the D0-D4/D8 holographic system of large $N_{c}$ QCD with non-trivial vacuum. Consequently, we can compute the $k$-body baryon potentials for arbitrary number of $k$ with this framework by considering the non-trivial QCD vacuum.

Our computation is parallel to [19] thus is straightforward. We took $k=3$ for the case of three baryons, i.e. the $U(3)$ matrix model and evaluate the Hamiltonian with a quantum three-body state which is a tensor product of single-baryon states. Then the potential intrinsic to the three-body case is obtained after subtracting the one- and two-body contributions. However our calculations are valid only at short distances, i.e. $1 / \sqrt{\lambda} M_{K K} \ll|r| \ll 1 / M_{K K}$ where $\lambda$ is the 't Hooft coupling constant9. As two typical and explicit examples, we choose (1) three-neutrons with averaged spins and (2) proton-proton-neutron (or proton-neutron-proton), and in both cases the baryons or nucleons are aligned on a line with equal spacings. We obtain the resultant three-body potentials for baryons in (3.56) and (3.60), both of which are positive (i.e. repulsive) and modified by the appearance of the smeared D0-branes (i.e. the non-trivial QCD vacuum). And as a quick check, all our results would return to [19] if setting $\zeta=0$, i.e. no smeared D0-branes.

Furthermore, we would like to give some more comments to our results and discuss the importance of them. According to the form of the wave function (2.9), our results (3.56) hold also for the case of threeprotons since the matrix action is $S U(2)$ invariance for isospin. So the results (3.56) hold if all three baryons or nucleons have the same flavor. Therefore, the three-body potential for proton-neutron-neutron takes the same form as (3.56), which implies the additional repulsive three-body force may exist in addition to twobody force and be affected by some effects from non-trivial QCD vacuum at short distances. Besides, the three-body potentials obtained in (3.56) and (3.60) are suppressed if compared with the two-body potential (2.10). At short distances, i.e. $1 / \sqrt{\lambda} M_{K K} \ll|r| \ll 1 / M_{K K}$, the suppression factor $1 / \lambda\left(r M_{K K}\right)^{2}$ is small which makes our computation valid.

On the other hand, as a difference from the original Sakai-Sugimoto model, we find the resultant threebody potentials (3.56) and (3.60) are totally complex if $\zeta>2$. It corresponds to the constraint for stable baryonic states in the D0-D4/D8 holographic system discussed in [8, 11] for two-body case. During our computation, the three-body potentials (3.56) and (3.60) actually come from the mass term in the matrix action (2.1) since the contributions from other terms vanish. From the matrix action (2.1), it is obvious to see that the matrix model describe an unstable system if $\zeta>2$ (i.e. a quantum mechanical system with complex mass term), that is the reason that the computation does not depend on the number density of the smeared D0-branes in the (3.14) from "classical treatment", while our results (3.56) and (3.60) are also consistent with these. Therefore according to our results in [8] and the three-body force (3.56) (3.60), it implies that the constraint for stable baryonic state may hold in the methods for $N$-body case. Besides, if comparing our results (3.56) and (3.60) with the two-body force (2.10) and (2.11), we find the three-body force would be going to infinity as $\zeta \rightarrow 1 / 2$. This implies the three-body force would become dominant if the non-trivial QCD vacuum is too important to be neglected, which is also different from [19].

With $\zeta<2$, these three-body forces would also become stronger if the distances get shorter. As a result, three-body forces give additional repulsive potential at short distances if the neutrons are highly dense. As mentioned, the effects from non-trivial QCD vacuum, for example in the glueball condensation or CME, may also play the important roles in such high-density matter. Physically, the mass spectrum of mesons is modified by considering the effects from non-trivial QCD vacuum with the D0-D4/D8 holographic system as

\footnotetext{
${ }^{9}$ If we fit pion decay constant with $\lambda$, then $M_{K K}$ would be $\mathcal{O}(1-0.5) \mathrm{GeV}$ when it fit with the mass of baryon or meson [16, 17, 24].
} 
discussed in [9], so the potential of the interaction among baryons would also be modified since the nucleons interact with each other by exchanging such mesons, which is also consistent with [8, 11]. In the viewpoint of

dual field theory, adding smeared D0-branes equals to add non-zero $\theta \operatorname{Tr}\left(F_{\mu \nu} \tilde{F}^{\mu \nu}\right)$ term to the action. With this term, the propagator derived in the dual quantum field theory is modified, thus yielding the modified three-body potential. Additionally, we also find the three-body forces for proton-proton-neutron and protonneutron-neutron are all positive i.e. repulsive, thus our result seems also responsible for Helium and Triton if the effects from the non-trivial vacuum are considered.

However, our results are as examples limited to three baryons on a line and only valid at short distances since the calculations for three baryons with generic positions are too messy to get the physical significance. So our result is not conclusive enough for those interests listed above but suggestive. Therefore, there is still a long way from holographic model with an underlying theory towards real-world nuclear matters.

\section{Acknowledgments}

This work is inspired by a seminar given by Chao Wu on his works 9, 11 and also as an extension to our previous works [8, 25, 26] in USTC. And we would like to thank Prof. Qun Wang and Dr. Chao Wu for helpful discussions.

\section{References}

[1] H. Leutwyler, Phys. Lett. B 96 (1980) 154; Nucl. Phys. B 179 (1981) 129..

[2] P. Minkowski, Nucl. Phys. B 177 (1981) 203..

[3] C. A. Flory, Phys. Rev. D 28 (1983) 1425.

[4] P. van Baal, Commun. Math. Phys. 94 (1984) 397

[5] G. V. Efimov, A. C. Kalloniatis, S. N. Nedelko, Phys. Rev. D 59 (1999) 014026 hep-th/9806165.

[6] J. Liao, "Chiral Magnetic Effect in Heavy Ion Collisions", arXiv:1601.00381.

[7] D. E. Kharzeev, J. Liao, S. A. Voloshin, G. Wang, "Chiral Magnetic Effect in High-Energy Nuclear Collisions - A Status Report", arXiv:1511.04050.

[8] S. Li, T. Jia, "Matrix model and Holographic Baryons in the D0-D4 background", Phys. Rev. D 92 (2015) 046007, arXiv:1506.00068.

[9] C. Wu, Z. Xiao, D. Zhou, "Sakai-Sugimoto model in D0-D4 background", Phys.Rev.D.88 (2013) 026016.

[10] K. Suzuki, "D0-D4 system and QCD_\{3+1\}”, Phys.Rev. D63 (2001) 084011, arXiv:hep-th/0001057.

[11] W. Cai, C. Wu, Z. Xiao, "Baryons in the Sakai-Sugimoto model in the D0-D4 background", Phys.Rev. D90 (2014) 106001, arXiv:1410.5549.

[12] K. Hashimoto, N. Iizuka, P. Yi, "A Matrix Model for Baryons and Nuclear Forces" , JHEP 10 (2010) 003 [arXiv:1003.4988]. 
[13] J. M. Maldacena, "The large N limit of superconformal field theories and supergravity", Adv. Theor. Math. Phys. 2, 231 (1998) [Int. J. Theor. Phys. 38, 1113 (1999)] arXiv:hep-th/9711200.

[14] S. S. Gubser, I. R. Klebanov and A. M. Polyakov, "Gauge theory correlators from non-critical string theory", Phys. Lett. B 428, 105 (1998) arXiv:hep-th/9802109].

[15] E. Witten, "Anti-de Sitter space and holography", Adv. Theor. Math. Phys. 2, 253 (1998) arXiv:hep-th/9802150.

[16] T. Sakai, S. Sugimoto, "Low energy hadron physics in holographic QCD", Prog. Theor. Phys. 113, 843 (2005) arXiv:hep-th/0412141.

[17] T. Sakai, S. Sugimoto, "More on a holographic dual of QCD", Prog. Theor. Phys. 114, 1083 (2005) arXiv:hep-th/0507073.

[18] E. Witten, "Baryons and branes in anti de Sitter space", JHEP 9807, 006 (1998) arXiv:hep-th/9805112.

[19] K. Hashimoto, N. Iizuka, "Three-Body Nuclear Forces from a Matrix Model", JHEP 11 (2010) 058, arXiv:1005.4412.

[20] K. Hashimoto, N. Iizuka, T. Nakatsukasa, "N-Body Nuclear Forces at Short Distances in Holographic QCD", Phys. Rev. D 81 (2010) 6003, arXiv:0911.1035.

[21] M. F. Atiyah, N. J. Hitchin, V. G. Drinfeld, Yu. I. Manin, "Construction of instantons", Phys. Lett. A 65,185 (1978).

[22] K. Hashimoto, T. Sakai, S. Sugimoto, "Nuclear Force from String Theory" , Prog. Theor. Phys. 122 (2009) 427-476, arXiv:0901.4449].

[23] N. H. Christ, E. J. Weinberg, N. K. Stanton, "General self-dual Yang-Mills solutions," Phys. Rev. D 18 (1978) 2013.

[24] H. Hata, T. Sakai, S. Sugimoto, S. Yamato, "Baryons from instantons in holographic QCD", Prog. Theor. Phys. 117, 1157 (2007), arXiv:hep-th/0701280.

[25] S. Li, A. Schmitt, Q. Wang, "From holography towards real-world nuclear matter", Phys.Rev. D92 (2015) 026006, arXiv:1505.04886.

[26] S. Li, "Baryon Transition in Holographic QCD", arXiv:1509.06914. 